OPEN ACCESS

Edited by:

Sandra Blaess,

University of Bonn, Germany

Reviewed by:

Fernando Giraldez,

Universitat Pompeu Fabra, Spain

Raj Awatramani,

Northwestern University,

United States

*Correspondence:

Mark Zervas

mark_zervas@brown.edu

Received: 26 March 2017

Accepted: 19 July 2017

Published: 22 August 2017

Citation:

Brown S and Zervas M (2017) Temporal Expression of Wnt1 Defines the Competency State and Terminal Identity of Progenitors in the Developing Cochlear Nucleus and Inferior Colliculus.

Front. Neuroanat. 11:67. doi: 10.3389/fnana.2017.00067

\section{Temporal Expression of Wnt1} Defines the Competency State and Terminal Identity of Progenitors in the Developing Cochlear Nucleus and Inferior Colliculus

\author{
Stephen Brown ${ }^{1}$ and Mark Zervas ${ }^{1,2,3 *}$ \\ ' Department of Molecular Biology, Cell Biology and Biochemistry, Division of Biology and Medicine, Brown University, \\ Providence, RI, United States, ${ }^{2}$ Department of Neuroscience, Division of Biology and Medicine, Brown University, \\ Providence, RI, United States, ${ }^{3}$ Department of Neuroscience, Amgen, Cambridge, MA, United States
}

The auditory system contains a diverse array of interconnected anatomical structures that mediate the perception of sound. The cochlear nucleus of the hindbrain serves as the initial site of convergence for auditory stimuli, while the inferior colliculus of the midbrain serves as an integration and relay station for all ascending auditory information. We used Genetic Inducible Fate Mapping (GIFM) to determine how the timing of Wnt1 expression is related to the competency states of auditory neuron progenitors. We demonstrate that the Wnt1 lineage defines progenitor pools of auditory neurons in the developing midbrain and hindbrain. The timing of Wnt1 expression specifies unique cell types during embryogenesis and follows a mixed model encompassing a brief epoch of de novo expression followed by rapid and progressive lineage restriction to shape the inferior colliculus. In contrast, Wnt1 fate mapping of the embryonic hindbrain revealed de novo induction of Wnt1 in auditory hindbrain progenitors, which is related to the development of biochemically distinct neurons in the cochlear nucleus. Thus, we uncovered two modes of lineage allocation that explain the relationship between the timing of Wnt1 expression and the development of the cochlear nucleus and the inferior colliculus. Finally, our analysis of Wnt1sw/sw mutant mice demonstrated a functional requirement of Wnt1 for the development of auditory midbrain and hindbrain neurons. Collectively, our study provides a deeper understanding of Wnt1 lineage allocation and function in mammalian brain development.

Keywords: Genetic Inducible Fate Mapping (GIFM), Wnt1, cell fate, inferior colliculus, cochlear nucleus, auditory nervous system

\section{INTRODUCTION}

The central auditory system is comprised of several anatomically distinct brain regions that are essential for normal sound processing. The innervation at multiple anatomical levels from brainstem to midbrain, and then to subcortical and cortical areas is vital for processing auditory information (Winer and Schreiner, 2005). The cochlear nucleus of the hindbrain receives topographically organized primary inputs from the cochlea of the inner ear (Osen, 1970). Neurons 
within the cochlear nucleus subsequently innervate the inferior colliculus (Cant and Benson, 2003; Malmierca et al., 2005), which then processes and integrates ascending information that is ultimately relayed to auditory centers in the thalamus and cerebral cortex (Aitkin and Phillips, 1984). Interestingly, the innervation and organization of the auditory hindbrain and midbrain are largely established before the onset of hearing (Friauf and Kandler, 1990; Kandler and Friauf, 1993; Gurung and Fritzsch, 2004). These findings suggest that developmental, but perhaps not activity-dependent, mechanisms play a primary role in the functional organization of the auditory system. The functional coordination between auditory centers is dependent upon the proper allocation and distribution of specific cell types during development. Concurrent with establishing mature neuronal phenotypes within the cochlear nucleus is the differential expression of the calcium-binding proteins parvalbumin (PV), calretinin (CALR), and calbindin (CALB) (Fredrich et al., 2009). However, it is unresolved how biochemically diverse neurons in the mature auditory system are established during development.

Cell fate specification of progenitors is likely to be involved in generating the diverse array of auditory neurons and the circuits they form. Neural progenitors have distinct, yet dynamic gene expression patterns which elicit unique functions at specific developmental time points (see for example Yang et al., 2013). One gene that is required for the development of the midbrain and hindbrain is Wnt1 (Wilkinson et al., 1987; McMahon and Bradley, 1990; McMahon et al., 1992; Ellisor et al., 2012; Yang et al., 2013). We used Genetic Inducible Fate Mapping (GIFM) (Joyner and Zervas, 2006) to mark Wnt1-expressing progenitors with fine temporal resolution and followed their fate and terminal identity to better understand how Wnt 1 is related to the development of auditory structures. Previous fate mapping studies have demonstrated that Wnt1expressing progenitors in the lower rhombic lip (LRL) of the anterior rhombencephalon and the mesencephalon contribute to the cochlear nucleus and inferior colliculus, respectively (Zervas et al., 2004; Farago et al., 2006; Nichols and Bruce, 2006). However, an important gap in the field of auditory system development has been determining the relationship between the timing of gene expression in progenitors and the terminal fate and spatial location of mature auditory neurons. Our analysis shows that the timing of Wnt1 expression in auditory progenitors predicts the extent, distribution, and molecular profile of mature neurons. GIFM coupled with marker analysis suggests a role for Wnt1 in the development of auditory circuits, which link the hindbrain and midbrain. From our findings, we propose two working models based on the competence model originally proposed by Livesey and Cepko (2001), to explain Wnt1-based cell lineage contribution to auditory structures. It should be noted that these models (lineage restriction and de novo expression) are not mutually exclusive.

Wnt1 encodes a secreted glycoprotein that plays diverse roles in development, including proliferation and cell fate decisions (Lee et al., 2004; Zervas et al., 2004; Ciani and Salinas, 2005; Brown et al., 2011; Hagan and Zervas, 2012;
Dingle et al., 2016; Hagan et al., 2017). Wnt1 is expressed in the rhombencephalon and mesencephalon, which, respectively, give rise to the hindbrain and midbrain (Wilkinson et al., 1987; Zervas et al., 2004; Farago et al., 2006). Multiple genetic strategies have uncovered functional roles of Wnt1 during development in vivo: (1) Targeted deletion of Wnt1 results in perinatal lethality and a failure of the midbrain and cerebellum to develop (McMahon and Bradley, 1990; Thomas and Capecchi, 1990); (2) In contrast, conditional gene deletion reveals distinct temporal roles for Wnt1 in midbrain development (Yang et al., 2013); and (3) Mice homozygous for a naturally occurring hypomorphic mutation of Wnt1, the swaying allele $\left(W n t 1^{s w / s w}\right)$, live to adulthood and exhibit variability in developmental and patterning deficits of the midbrain and cerebellum (Bronson and Higgins, 1967; Lane, 1967; Thomas et al., 1991; Bally-Cuif et al., 1995; Ellisor et al., 2012). However, how the hindbrain and midbrain auditory centers are affected by the $W n t 1^{s w}$ allele has not been determined at a cellular level. Therefore, we used marker analysis to investigate how the cochlear nucleus and inferior colliculus are organized in $W n t 1^{s w / s w}$ mice. We found mild to severe perturbations of the inferior colliculus and a mild, but invariant phenotype of the cochlear nucleus of $W n t 1^{s w / s w}$ mice. Thus, this study provides a framework to investigate divergent and common mechanisms by which Wnt1 establishes the cochlear nucleus and inferior colliculus.

\section{MATERIALS AND METHODS}

\section{Mice and Genotyping}

Wnt1-Venus mice were generated by C. Bromleigh and gratefully obtained from A. Joyner (Memorial Sloan Kettering Cancer Center). This line was generated by subcloning a cassette encoding yellow fluorescent protein (Venus, a green fluorescent protein variant) into a multi-cloning site between the translated and untranslated region of exon 1, which places Venus under the control of Wnt1 regulatory elements. This same configuration was also used to control CreER ${ }^{T}$ expression in Wnt1-CreER ${ }^{T}$ transgenic mice (Zervas et al., 2004). The fidelity of this transgene has been verified by in situ hybridization (Brown et al., 2011). Our previously published studies have validated that the Wnt1-GFP and Wnt1-CreER ${ }^{T}$ lines used in this study recapitulate endogenous $W n t 1$ expression in both the midbrain and hindbrain. See for example: (1) Zervas et al. (2004) and (2) Brown et al. (2011), which show that Wnt1-YFP and Wnt1CreER ${ }^{T}$ mimics endogenous $W n t 1$ expression in the midbrain by whole mount labeling and see also (3) Supplementary Figure 1 of Ellisor et al. (2009) for comparison/validation between $W n t 1-C r e E R^{T}$ and $W n t 1$ in sagittal sections] and (4) Supplementary Figure 1 of Hagan and Zervas (2012) for comparison/validation between Wnt1-Venus and Wnt1 in sagittal sections and Supplementary Figure 2 of Hagan and Zervas (2012) for comparison/validation between Wnt1$C_{r e E R^{T}}$ and $W n t 1$ in sagittal sections. Reporter $m G F P$ mice (Tau lox-STOP-loxmGFP-IRES-NLS-LacZ-PA, Hippenmeyer et al., 2005) were generously provided by S. Arber, which allowed us 
to follow the Wnt1 lineage in vivo (For example see: Brown et al., 2009; Ellisor et al., 2009; Ellisor and Zervas, 2010; Hagan and Zervas, 2012). Mice were housed and handled in accordance with Brown University Institutional Animal Care and Use Committee guidelines. Genotyping was done as previously described (Brown et al., 2009; Ellisor et al., 2009). Wnt1-Venus embryos were detected by whole-mount fluorescence (Figure 1) using filters for GFP as previously shown (Ellisor et al., 2012). In tissue sections, the Wnt1-Venus transgene was detected with anti-GFP antibodies (described below).

\section{Embryonic Tissue Preparation}

Wnt1-Venus transgenic males were bred with Swiss Webster females (Taconic) to obtain litters at three different embryonic stages: E8.5, E10.5, and E12.5 (Figure 1). Embryos were dissected in PBS over ice and Wnt1-Venus embryos were identified by GFP fluorescence, imaged, and confirmed by genotyping for GFP
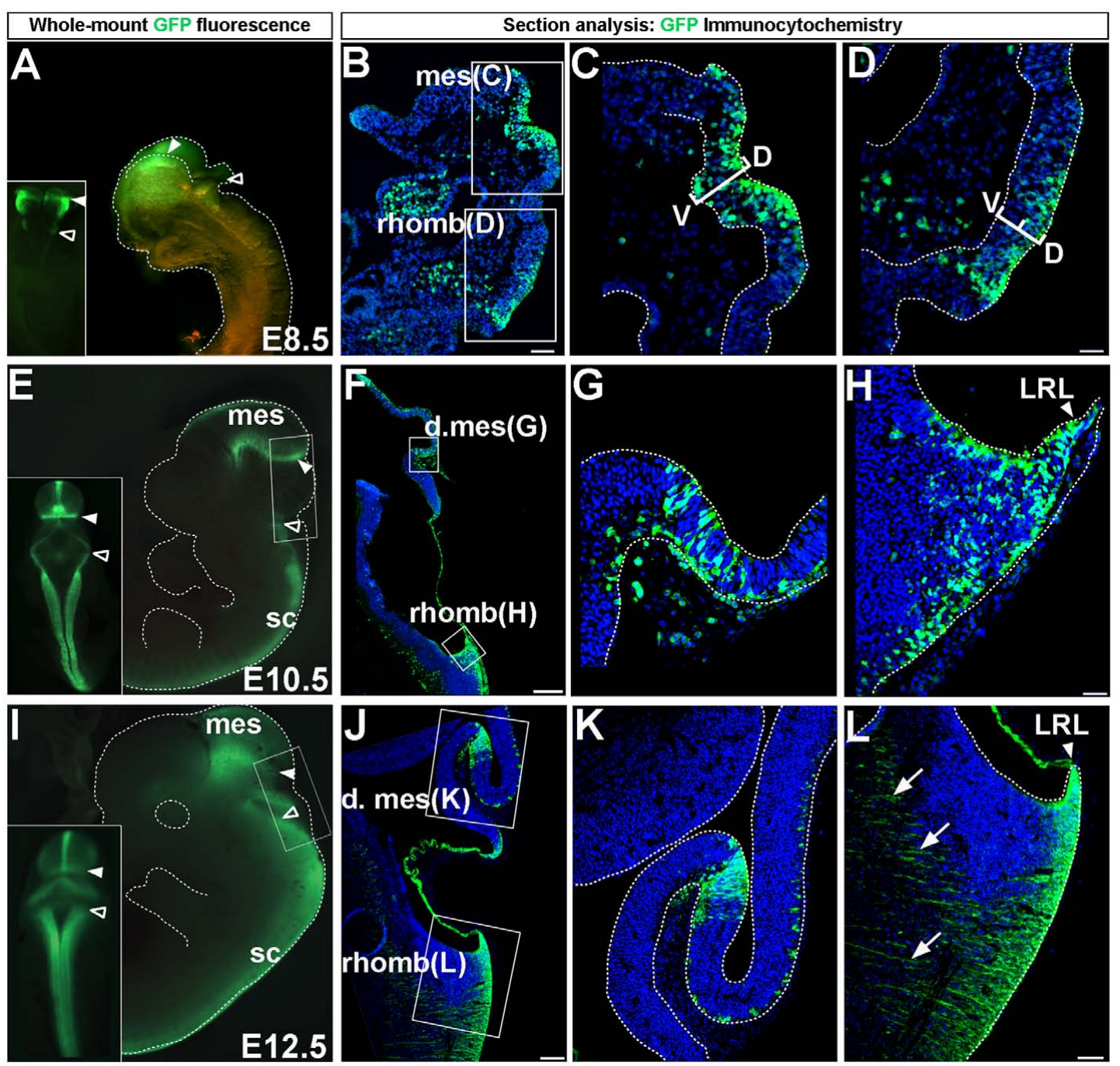

FIGURE 1 | Wnt1 expression in midbrain and hindbrain auditory primordia. Wnt1 expression observed by GFP whole-mount fluorescence and GFP immunocytochemistry in Wnt1-Venus transgenic embryos. (A-D) Expression of Wnt1 at E8.5. (A) Wnt1 was expressed diffusely throughout the mes (solid arrowhead) and was faintly observed in the rhomb (open arrowhead). (B-D) Sagittal sections show detailed Wnt1 expression within the neuroepithelium. Wnt1 was expressed through the D-V extent of the mesencephalic neuroepithelium (C, bracketed area) while faint expression in the rhomb was confined to the dorsal half of the neuroepithelium (D, bracketed area). (E-H) Wnt1 expression at E10.5. (E) Wnt1 was confined to a ring at the posterior limit of the dorsal mes (solid arrowhead) while Wnt1 in the rhomb was expressed throughout the LRL (open arrowhead). (F) A mid-sagittal section shows the spatial segregation of Wnt1 expression in the mes and rhomb. (G,H) High magnification shows GFP positive cells remaining within the neuroepithelium. (I-L) Wnt1 expression at E12.5. (I) The pattern of Wnt1 expression at E12.5 was diminished within the posterior ring in the dorsal mes (solid arrowhead) and was broadened within the LRL (open arrowheads). (J,K) Wnt1 in sagittal sections of the mes was restricted at E12.5 compared to the broader profile at E10.5. (L) The LRL showed broadened Wnt1 expression in the neuroepithelium at E12.5. We took advantage of the likely perdurance of GFP, which allowed the early tracking of precursors as they migrate out of LRL and began to form projections (arrows). Insets in (A,E,I) display whole-mount dorsal views. mes, mesencephalon; d. mes, dorsal mesencephalon; rhomb, rhombencephalon; sc, spinal cord; LRL, lower rhombic lip; D-V, dorsal-ventral. Scale bars: (B) $63 \mu \mathrm{m}$; (C,D) $32 \mu \mathrm{m}$; (F) $260 \mu \mathrm{m}$; (G,H) $32 \mu \mathrm{m}$; (J) $130 \mu \mathrm{m}$; (K,L) $63 \mu \mathrm{m}$. 
(Ellisor et al., 2009). Embryos were fixed in 4\% paraformaldehyde (PFA) overnight at $4^{\circ} \mathrm{C}$, cryoprotected, and embedded in OCT. Embryos ( $n>3$ across two litters for each embryonic stage) were sectioned sagittally $(12 \mu \mathrm{m})$ with a Leica cryostat and stored in ziplock bags at $-20^{\circ} \mathrm{C}$. Sections were immunolabeled as described below.

\section{Genetic Inducible Fate Mapping (GIFM)}

Genetic Inducible Fate Mapping experiments were conducted by crossing Wnt1-CreER ${ }^{T}$; $m$ GFP males with Swiss Webster wild type females (Taconic) as previously described (Brown et al., 2009; Ellisor et al., 2009; Ellisor and Zervas, 2010). The morning (0900) of the day a vaginal plug was detected was designated as 0.5 days post-coitus. Tamoxifen was administered at a dose of $4 \mathrm{mg}$ to time-pregnant females by oral gavage at 0900 (Brown et al., 2009; Ellisor et al., 2009). Note that descriptions of the initially marked populations of the Wnt1 lineage (labeled by tamoxifen administration at E8.5, E9.5, and E10.5) can be found in Zervas et al. (2004) (Figures 2B, 3B, 5E, inset, respectively). Mice were genotyped by obtaining a tail biopsy and performing PCR analysis on DNA from tail lysates. At 6 weeks of age Wnt1-CreER ${ }^{T} ; m G F P$ fate mapped mice were deeply anesthetized with Nembutal $(100 \mathrm{mg} / \mathrm{kg})$ and intracardially perfused with PFA. Craniotomies were performed to extract brains, which were stored in PFA at $4^{\circ} \mathrm{C}$ until sectioning. Brains were embedded in 3\% agarose in PBS and sectioned coronally $(40 \mu \mathrm{m})$ with a Leica vibratome (Brown et al., 2009). Fate mapped brains $(n>3)$ across two litters were processed for marking and analysis.

\section{Immunocytochemistry}

Sections were immunolabeled as previously described (Ellisor et al., 2009). Sagittal sections from Wnt1-Venus embryos at E8.5, E10.5, and E12.5 were analyzed using an anti-GFP antibody (1:600, Molecular Probes, Cat \# A-6455). Adult coronal sections for fate mapping experiments were immunolabeled with an anti- $\beta$-galactosidase $(\beta$-gal) antibody (1:500, Biogenesis, Cat \# 4600-1409 or 1:500, Abcam, Catalog \# ab9361-250) to identify Wnt1-derived cells (Ellisor et al., 2009). In addition, antiCalretinin (1:5000, Chemicon; Billerica, MA; Catalog \# AB1550), anti-Calbindin (1:1000, Swant, Catalog \# CB3a), and antiParvalbumin (1:1000, Sigma, Catalog \# P3088-.2ML) antibodies were used as biomarkers. Secondary antibodies were prepared at 1:500 and include: Alexa 488 (Invitrogen; Cat \# A-21206, donkey anti-rabbit IgG; Cat \# A-21202, donkey anti-mouse IgG; Cat \#A-11055 donkey anti-goat IgG), Dylight 549 (Jackson ImmunoResearch Laboratories; Cat \#703-505-155, donkey antichicken).

\section{Histology}

Silver staining was performed as previously described (Gallyas, 1979; See also Zervas and Walkley, 1999). Briefly, $40 \mu \mathrm{m}$ thick sagittal sections from $W n t 1^{+/+}$and $W n t 1^{s w / s w}$ mice (processed in parallel) were mounted on glass slides and incubated in pyridine:acetic anhydride (2:1) for $30 \mathrm{~min}$. Sections were rinsed three times in $\mathrm{dd}_{2} \mathrm{O}$ for $5 \mathrm{~min}$, followed by a $45 \mathrm{~min}$ incubation in ammoniacal silver nitrate. Sections were rinsed three times in $0.5 \%$ acetic acid for $10 \mathrm{~min}$, incubated in developer solution (Gallyas, 1979) for $5 \mathrm{~min}$, and terminated by incubating in 1\% acetic acid for $10 \mathrm{~min}$. Sections were dehydrated through ethanol followed by xylene. Slides were coverslipped with Permount mounting media.

\section{Microscopy and Cell Counting}

Whole-mount images were obtained with a Leica MZ16F stereoscopic epifluorescent microscope using PictureFrame3 software. Images of tissue sections were obtained with a Leica DM6000B epifluorescent microscope using Volocity 5.2 imaging software (Improvision). Low magnification images were captured with $2.5 \times$ and $5 \times$ objectives while high magnification images were obtained using a motorized stage with $10 \times$ and $20 \times$ objectives. Actual magnifications are indicated in figures by scale bars. All images were pseudo colored live as part of the acquisition palettes. Imaging data sets were exported to Adobe Photoshop CS3 where montages of representative data were generated. We counted neurons in the central nucleus of the inferior colliculus by acquiring $10 \times$ images spanning the entire central nucleus that were stitched together in Adobe Photoshop CS3. Profiles in the red channel corresponded to nuclear $\beta$-gal labeling of fate mapped neurons in accordance with the genetics of our fate mapping alleles (Brown et al., 2009; Ellisor et al., 2009; Ellisor and Zervas, 2010). Images were imported into Image J and data was converted to binary images and counted using the Analyze Particles function. Magnocellular neurons of the cochlear nucleus were manually counted to avoid the densely labeled granule cell layers that were prohibitive to the automated method described above. Manual counting was done by analyzing images in Photoshop CS3 with the Count Tool function. For each time point, three representative sections from three separate animals (nine sections total per time point) were counted for each structure. Data are represented as the average number ( \pm the standard deviation) of $W n t 1$-derived neurons per section. Statistical analysis using Student's $t$-test was performed to determine the significance of change in contribution between time points of analysis.

\section{RESULTS}

\section{Wnt1 Expression in Auditory Primordia}

Prior to fate mapping the Wnt1 lineage, we assessed the distribution of Wnt1-expressing progenitors in Wnt1-Venus embryos (Brown et al., 2011) to demonstrate where the initial populations of midbrain and hindbrain progenitors resided at key developmental stages. We evaluated GFP-labeled domains by whole-mount fluorescence and GFP immunocytochemistry at embryonic day (E)8.5, E10.5, and E12.5 (Figure 1). The expression patterns described below for the Wnt1-Venus transgenic line reflects Wnt1 expression as detected by in situ hybridization (See Materials and Methods, Mice and Genotyping for details and references). Wnt1 delineated the mesencephalon and was faintly observed in more posteriorly located rhombomeres at E8.5 (Figure 1A) consistent with in situ hybridization experiments (Wilkinson et al., 1987; Zervas 


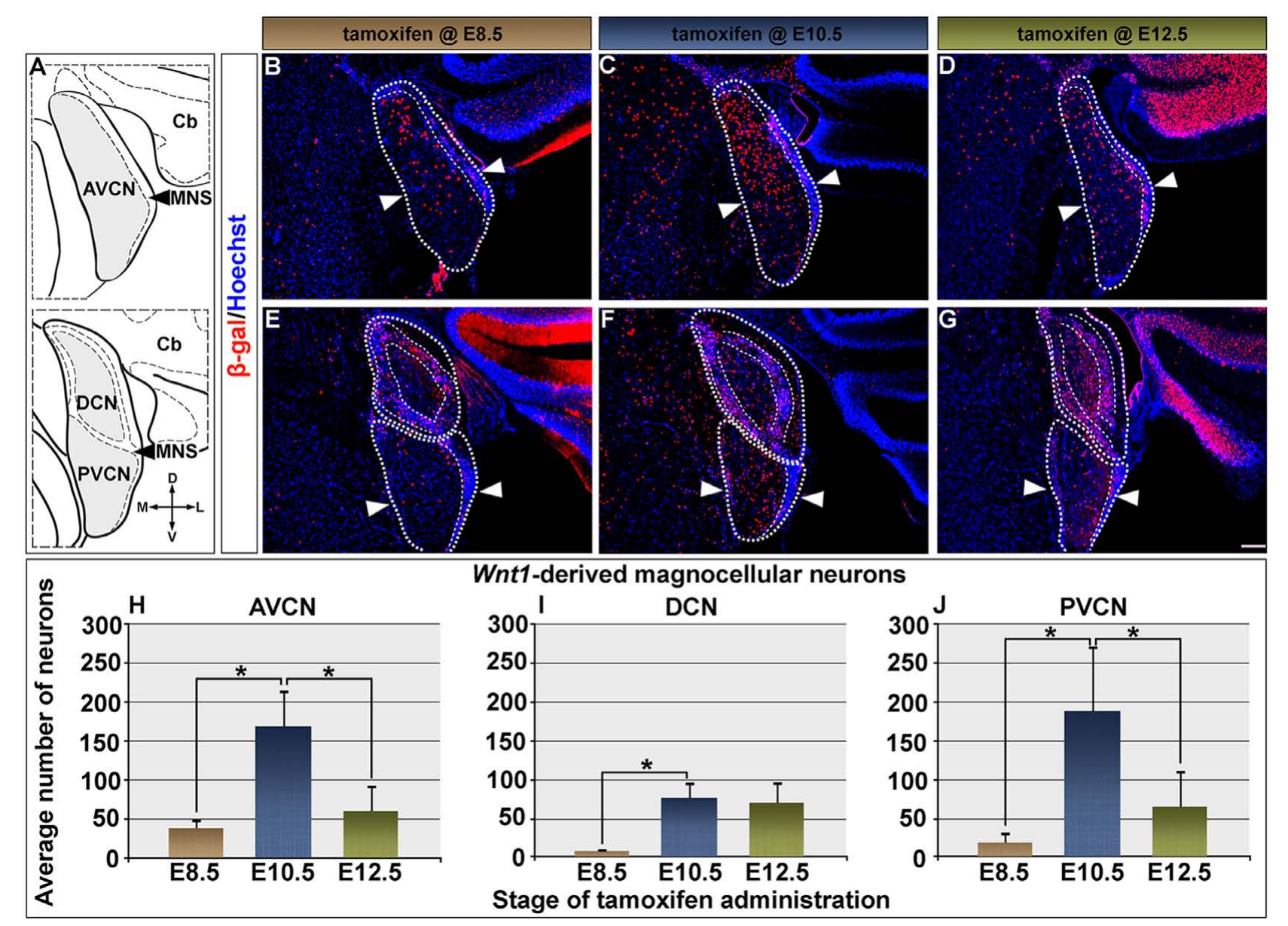

FIGURE 2 | Timing of Wnt1 expression in predicts the extent and distribution of neurons within the cochlear nucleus. (A) Schematic representation of the AVCN, DCN, and PVCN in coronal planes. Areas shaded in gray delineate the magnocellular core (MGC) of each nucleus, which was counted. Fine dashed lines represent the borders of the microneuronal shell (MNS); the laterally located cerebellum is denoted as Cb. Wht1-derived neurons labeled by immunocytochemistry in the rest of the figure were nuclear $\beta$-gal+ (red). (B-D) The contribution of Wnt1-expressing progenitors to the AVCN are show: (B,H) Progenitors marked at E8.5 were predominantly found in a dorsal domain of the AVCN (above the line delineated by arrowheads) and yielded $38.9 \pm 12.0$ Wnt1-derived neurons/section. (C,H) Wnt1-expressing progenitors marked at E10.5 were dorsally distributed in the mature AVCN and gave rise to $169.4 \pm 47.2$ Wnt1-derived neurons/section. (D,H) By E12.5, Wnt1-expressing progenitors ultimately contributed to the AVCN in decreased numbers (60.8 \pm 34.4 Wnt1-derived neurons/section). (E-G) Magnocellular neurons of the DCN and PVCN were derived from progenitors with late Wnt1 expression. (E,I) DCN progenitors which expressed Wnt1 at E8.5 largely contributed to the MNS, with few Wnt1-derived MGC neurons (8.0 \pm 4.1 Wnt1-derived neurons/section). (F,I) Wnt1-expressing progenitors marked at E10.5 gave rise to DCN neurons throughout the D-V axis of the MGC and continued to populate the MNS (75.9 \pm 19.9 Wnt1-derived neurons/section). (G,I) The contribution of Wnt1-expressing progenitors to DCN MGC neurons continued through E12.5 (70.2 \pm 27.8 Wnt1-derived neurons/section). (E-G) Wnt1 derived neurons populated the PVCN with a similar profile as the AVCN. (E,J) Wnt1-expressing progenitors marked at E8.5 contributed sparsely to the dorsal aspects of the PVCN (18.3 \pm 14.4). (F,J) Peak contribution of Wnt1-expressing progenitors (188.4 \pm 85.0) to the PVCN arose from E10.5 expression. (G,J) In contrast, Wnt1-expressing progenitors marked at E12.5 (65.1 + 46.4) contributed in fewer numbers throughout the D-V axis. Asterisks denote average numbers of Wnt1-derived neurons, which were significantly different $(p<0.005)$. Scale bars: $130 \mu \mathrm{m}$ (B-G).

et al., 2004). We also analyzed mid-sagittal sections to provide a more detailed description of Wnt1 at E8.5 (Figures 1B-D). Wnt1 spanned the dorsal-ventral (D-V) extent of the mesencephalic neuroepithelium (Figures 1B,C, bracketed area). However, Wnt1 was heterogeneously expressed and progenitors not expressing Wnt1 were also observed within the mesencephalon expression domain (Figure 1C). Wnt1 in the rhombencephalon was restricted to the dorsal tier of the neuroepithelium (Figures 1B,D, bracketed area). At E10.5, whole mount fluorescence revealed that Wnt 1 had become confined to the dorsal and ventral midline and to a ring of expression at the posterior limit of the dorsal mesencephalon (Figure 1E, solid arrowhead), in agreement with previous reports (Wilkinson et al., 1987; Gavin et al., 1990; Ellisor et al., 2009). In comparison to E8.5, Wnt1 had expanded in the LRL at E10.5 (Figure 1E, open arrowhead). Analysis of Wnt1 at E10.5, in midline sagittal sections, clarified the posterior expression in the mesencephalon (Figures 1F,G), as well as the broadened expression throughout the LRL (Figures 1F,H). Wnt1expressing progenitors in the neuroepithelium of the dorsal mesencephalon at E10.5 appeared clonal and were interspersed 
amongst non-expressing progenitors (Figure 1G). Similarly, progenitors that did not express Wnt1 were found within the LRL at E10.5 (Figure 1H). The general whole-mount pattern of expression observed at E10.5 persisted until E12.5 (Figure 1I), although the lateral aspects of the posterior ring of the mesencephalon showed diminished expression (Figure 1I, solid arrowheads), while expression in the LRL continued to broaden (Figure 11, open arrowheads). Analysis of sagittal sections confirmed that the expression domain was tightly restricted in the posterior mesencephalon at E12.5 (Figures 1J,K). Expression of Wnt1 was also observed in the LRL at E12.5 (Figures 1J,L) and consisted of early projections (Figure 1L, arrows), which contributed to the broadened expression domain, as well as cells emanating from the LRL. Wnt1-GFP expression at E12.5 may exhibit perdurance of GFP, which allowed us to observe precursors as they migrated out of LRL and formed projections (Figure 1L, arrows). Note that the possibility of GFP perdurance could confound our assessment of the precursors expressing $W n t 1$ at this stage. However, we provided representative data and rendered a fair interpretation of Wnt1-expressing progenitors.

\section{The Wnt1 Lineage Contributes to the Cochlear Nucleus in Distinct Temporal Windows}

Next, we addressed how Wnt1-expressing progenitors in the rhombencephalon contributed to the mature auditory hindbrain using GIFM (Joyner and Zervas, 2006). Specifically, we used Wnt1-CreER ${ }^{T} ; m G F P$ transgenic mice bred to Swiss Webster females to determine how the timing of Wnt1 expression in progenitors was related to the organization of the cochlear nucleus. We administered tamoxifen to time-pregnant females between E8.5 and E14.5 and analyzed the cochlear nucleus at 6 weeks of age. The expression of the Wnt1-CreER ${ }^{T}$ transgene accurately reflect Wnt1 expression as detected by in situ hybridization (See Materials and Methods, Mice and Genotyping for details). In addition, a description of the initially marked populations of the Wnt1 lineage (labeled by tamoxifen administration at E8.5, E9.5, and E10.5) can be found in Zervas et al. (2004) (Figures 2B, 3B, 5E, inset, respectively).

Here, we used the $m G F P$ reporter, which is driven by the Tau locus and allowed us to strictly assess the Wnt1 lineage contribution to neurons (Hippenmeyer et al., 2005). Neuronal descendants of progenitors that expressed Wnt1 (at the time of tamoxifen administration) continue to express nuclear $\beta$-galactosidase $(\beta$-gal) and were identified through immunocytochemistry (Figures 2-5). The hindbrain cochlear nucleus is partitioned into three distinct divisions with overlapping function and organization: the anteroventral cochlear nucleus (AVCN), dorsal cochlear nucleus (DCN), and the posteroventral cochlear nucleus (PVCN) (Ryugo and Parks, 2003). The Wnt1 lineage contributed to neurons within subdivisions of each of the cochlear nuclei over a broad developmental window (marking from E8.5 to E12.5) (quantified in Figure 2). The distribution pattern of $W n t 1$-derived neurons differed amongst the individual nuclei and was dependent on the time that progenitors expressed $W n t 1$. AVCN progenitors in the
LRL, which express Wnt1 from E8.5 through E12.5 (Figure 1), gave rise to magnocellular neurons distributed primarily in the dorsal aspect of the AVCN with the peak contribution occurring at E10.5 (Figures 2B-D). However, Wnt1-expressing progenitors marked at E10.5 and E12.5 also contributed to neurons positioned in the ventral domain of the AVCN resulting in a more even distribution throughout the D-V axis (Figures 2C,D). Interestingly, granule cells within the microneuronal shell (MNS) of the AVCN were derived in greater abundance from progenitors that expressed Wnt1 at E10.5, with the peak contribution at E12.5 (Figures 2B-D, compare the MNS, which is denoted in Figure 2A). In contrast, there was not a discernible D-V bias in the distribution of Wnt1-derived magnocellular neurons of the DCN at any marking stage (Figures 2E-G). Sparsely labeled neurons derived from Wnt1-expressing progenitors at E8.5 were distributed in the dorsal half of the PVCN (Figure 2E). Wnt1-expressing progenitors marked at E10.5 resulted in the peak contribution to the PVCN while progenitors marked at E12.5 contributed to neurons distributed throughout the lateral core of the PVCN and its MNS (Figures 2F,G).

We quantified the contribution of Wnt1-expressing progenitors to magnocellular neurons within the cochlear nuclei (Figures 2H-J). Magnocellular neurons of the AVCN were predominantly derived from progenitors expressing Wnt1 at E10.5 (169.4 \pm 47.2 Wnt1-derived neurons/section), which was a fourfold increase compared to marking at E8.5 (38.9 \pm 12.0 Wnt1-derived neurons/section) (Figure 2H). Despite extensive Wnt1 expression observed in the developing hindbrain at E12.5, the contribution of the Wnt1 lineage to AVCN neurons declined to $60.8 \pm 34.4$ Wnt1-derived neurons/section (Figure $2 \mathbf{H}$ ). By E14.5, progenitors expressing Wnt1 no longer contributed to the cochlear nucleus (data not shown). In contrast to the AVCN, very few neurons within the DCN were derived from Wnt1-expressing progenitors marked at E8.5 (8.0 \pm 4.1 Wnt1-derived neurons/section) (Figure 2I). Unlike the sharp peak of contribution observed in the AVCN when marked at E10.5, the contribution to the DCN was more limited and occurred over an extended time period (from E10.5 to E12.5) (Figure 2I). The number of DCN neurons derived from the Wnt1 lineage marked at E10.5 and E12.5 were not significantly different $(75.9 \pm 19.9$ compared to $70.2 \pm 27.8$ Wnt1-derived neurons/section, respectively) (Figure 2I). The contribution of Wnt1-expressing progenitors to PVCN neurons resembled that of the AVCN with a markedly sharp peak of contribution to the magnocellular core when marked at E10.5 (Figure 2J). Specifically, there was a 10 -fold increase in the contribution of the Wnt1 Lineage to the PVCN when comparing marking at E10.5 (188.4 \pm 85.0 Wnt1-derived neurons/section) versus marking at E8.5 (18.3 \pm 14.4$)$ (Figure 2J). Although there was broad Wnt1 expression in the hindbrain at E12.5, the contribution of the Wnt1 lineage to PVCN neurons significantly decreased when marked at this stage (65.1 \pm 46.4 Wnt1-derived neurons/section) (Figure 2J). In summary, Wnt1-expressing progenitors marked at E8.5 gave rise to neurons in cochlear nuclei with labeled neurons in the dorsal AVCN/PVCN or the MNS of the DCN. The Wnt1 lineage marked at E10.5 resulted in the peak of contribution to AVCN/PVCN neurons. In contrast, 
there was prolonged, but less substantial contribution of the Wnt1 lineage to DCN neurons from E10.5 to E12.5. Notably, the early (E8.5) expression of Wnt1 did not produce Wnt1-derived neurons being evenly distributed throughout the AVCN, DCN, nor PVCN of the cochlear nucleus, which suggests that de novo Wnt1 expression occurred in progenitors to generate the full complement of the Wnt1 lineage contribution to the auditory hindbrain. These findings suggest that the timing of Wnt1 is related to the specification of distinct cell types in the cochlear nucleus.

\section{Temporally Labeled Wnt1-Expressing Progenitors Contribute to Biochemically Distinct Neurons in the Cochlear Nucleus}

The calcium-binding proteins PV, CALB, and CALR are expressed exclusively in neurons (Bredderman and Wasserman, 1974; Heizmann, 1984; Rogers, 1987; Celio, 1990). Moreover, the expression of PV, CALB, and CALR correlates with the functional distribution of neurons in cochlear nuclei of rat (Fredrich et al., 2009). We used these calcium-binding proteins as molecular markers to determine whether the timing of Wnt1 expression was related to the establishment of biochemically distinct neurons of the cochlear nucleus. We did this by performing double immunocytochemistry to identify how the Wnt1 lineage ( $\beta$-gal positive, red) contributed to PV, CALB, or CALR (green) neurons in the AVCN, DCN, and PVCN (Figures 3-5).

Parvalbumin-positive neurons in the magnocellular core of the AVCN were derived from the Wnt1 lineage across all stages of marking (Figures 3A-C, insets). These findings show that Wnt1-expressing progenitors contributed to the PV-positive subpopulation in the AVCN, likely inclusive of T-stellate and spherical-bushy, but not globular-bushy neurons (Pór et al., 2005; Fujiyama et al., 2009). Notably, CALB+ and CALR+ neurons in the AVCN were not derived from the Wnt1 lineage at any marking stage (Figures 3D-I).

Parvalbumin-expressing neurons in the DCN, likely MLstellate cells (Caicedo et al., 1996; Fujiyama et al., 2009), were not derived from progenitors expressing Wnt1 at any time point examined (Figures 4A-C). In contrast, CALB-positive neurons in the DCN, that is fusiform and cartwheel cells (Frisina et al., 1995), were derived from progenitors expressing Wnt1, but only sparsely and at E10.5 and E12.5 (Figures 4D-F, insets). CALRpositive neurons in the DCN, likely unipolar brush cells (Floris et al., 1994; Di Bonito and Studer, 2017), were derived from the Wnt1 Lineage, but only at E12.5 (Figures 4G-I).

Similar to the AVCN, Wnt1-expressing progenitors marked from E8.5 to E12.5 gave rise to PV-positive neurons in the PVCN, which is inclusive of T-stellate cells (Di Bonito and Studer, 2017) (Figures 5A-C). CALB-positive neurons of the PVCN, including putative octopus cells (Di Bonito and Studer, 2017), were also derived from the Wnt1 lineage at each marking stage (Figures 5D-F), which was distinctly different compared to the AVCN. CALR+ neurons in the PVCN were not derived from the Wnt1 lineage as any marking stage (Figures 5G-I). In summary, neurons expressing calcium binding proteins, which render these cohorts as functionally distinct neuronal subtypes, were derived from the Wnt1 lineage in temporal patterns unique to each subdivision in the cochlear nucleus.

\section{The Auditory Midbrain Is Established through Progressive Lineage Restriction after E9.5}

We next determined how Wnt1-expressing progenitors in the mesencephalon contributed to the auditory midbrain using GIFM, as described above for the cochlear nucleus. Wnt1-expressing progenitors at E8.5 (Figure 1A, solid arrowheads) were marked and assed for their contribution and distribution in the adult midbrain (schematic view shown in Figure 6A). Marking at E8.5 resulted in neurons being distributed throughout the inferior colliculus (Figure 6B). Quantitative analysis showed that progenitors marked at E8.5 gave rise to $919.3 \pm 307.5$ Wnt1-derived neurons/section (Figure 6E). Marking at E9.5, which is subsequent to neural tube closure, resulted in a substantial peak of labeled neurons contributing to the inferior colliculus in adulthood (Figure 6C). Specifically, we observed a fourfold increase in the number of marked neurons at E9.5 (3923.8 \pm 722.7 Wnt1-derived neurons/section) compared to marking a day earlier (Figure 6E). The large increase in contribution indicates that de novo expression of Wnt1 occurred as the mesencephalon underwent morphometric changes likely necessary for neural tube closure. Despite the preserved Wnt1 expression domain at E10.5, there was a rapid and large decline in the lineage contribution (181.7 $\pm 132.8 W n t 1$-derived neurons/section) to the inferior colliculus marked at this stage (Figures 6D,E). Wnt1-expressing progenitors contributed to $\mathrm{PV}$-expressing neurons, which were found throughout each of the inferior colliculus subdivisions (Coleman et al., 1992; Vater and Braun, 1994; Lohmann and Friauf, 1996; Paloff et al., 2004; Sharma et al., 2009) (insets in Figures 6B-D).

Marking the Wnt1 lineage at E8.5 or E9.5 resulted in cells being distributed along the rostral-caudal axis of the inferior colliculus with the most substantial contribution occurring at E9.5 (Figures 7A-D). The distribution of the Wnt1 lineage marked at E10.5 was largely confined to the dorsomedial aspects of the anterior (rostral) inferior colliculus while the most significant contribution occurred in the posterior domain of the inferior colliculus (Figures 7E,F). In contrast, marking at E11.5 did not yield appreciable contribution to the inferior colliculus proper anteriorly and resulted in a significant decline in contribution to the posterior inferior colliculus (Figures $7 \mathbf{G}, \mathbf{H}$ ). Collectively, GIFM revealed the differential contribution of the Wnt1 lineage marked at specific time points (described above) to the auditory hindbrain and midbrain, which is summarized in Figure 8.

\section{Wnt1 Is Functionally Required for Establishing Hindbrain and Midbrain Auditory Centers}

We took advantage of mice homozygous for the Swaying allele $\left(W n t 1^{s w / s w}\right)$ to investigate how a mutation in Wnt1 alters 


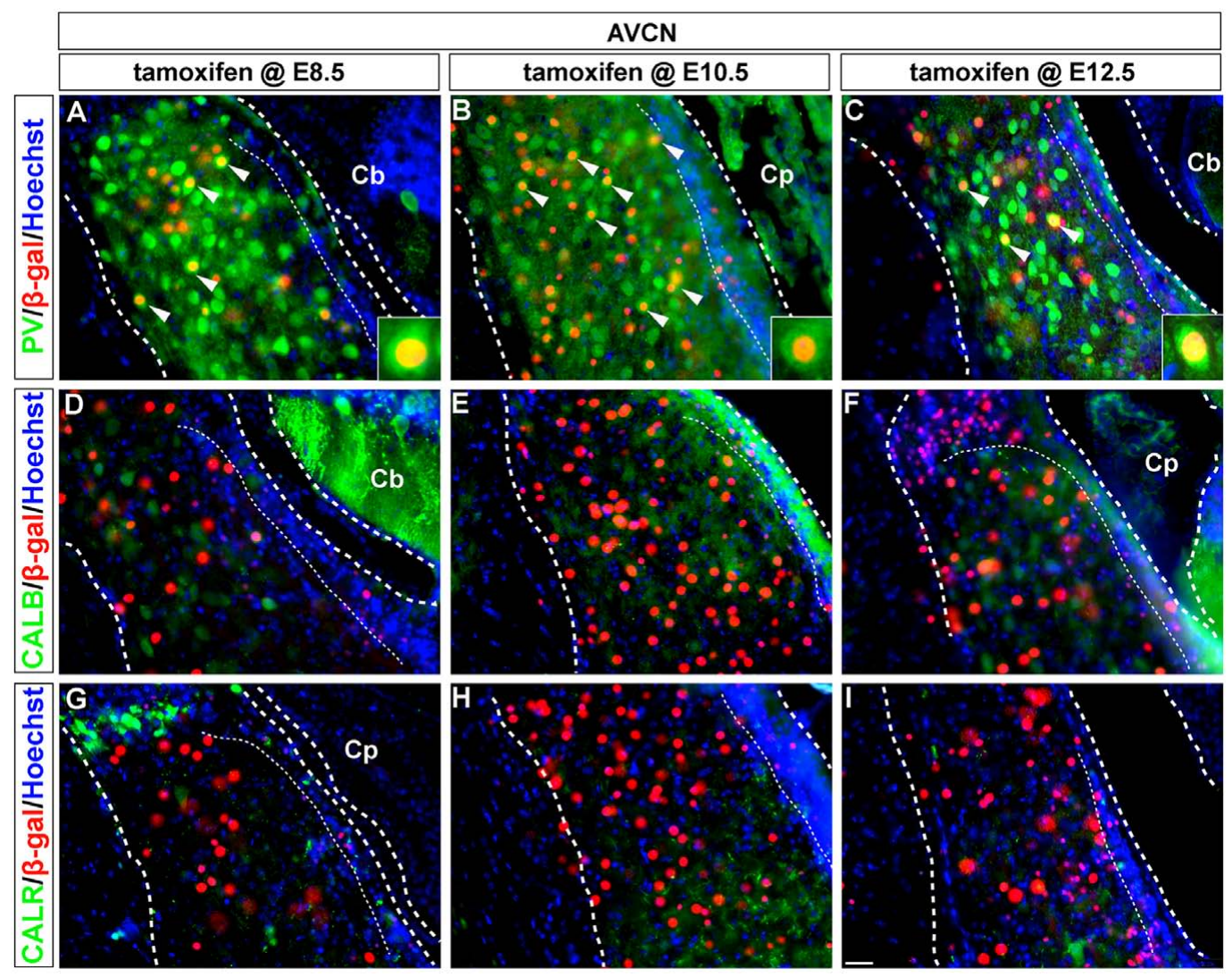

FIGURE 3 | PV-expressing AVCN neurons are derived from Wnt1-expressing progenitors. (A-I) Wht1-derived neurons in the AVCN were identified by nuclear $\beta$-gal immunocytochemistry (red) and with indicated biochemical markers (green). Wnt1 marking between E8.5 and E12.5 defined biochemically distinct neurons of the AVCN, which expressed PV (A-C, arrowheads, insets). Neither CALB (D-F) nor CALR (G-I) expressing neurons neurons in the AVCN were derived from Wnt1-expressing progenitors between E8.5 and E12.5. Broad dashed lines indicate the AVCN structure, while fine dashed lines delineate the granule cell layer. Cb, cerebellum; Cp, choroid plexus. Scale bar in (I) indicates $32 \mu \mathrm{m}$.

the development of the cochlear nucleus (hindbrain) and the inferior colliculus (midbrain). The swaying allele is a naturally occurring single base pair deletion in the coding sequence of Wnt1 that is believed to result in truncated WNT1 protein with hypomorphic function (Thomas et al., 1991). Wnt ${ }^{s w / s w}$ mice display variable gross morphological phenotypes of the midbrain and cerebellum (Thomas et al., 1991; Ellisor et al., 2012) (Figure 9).

However, the degree to which the cell types of the auditory midbrain and hindbrain are affected in $W n t 1^{s w / s w}$ mice has not been reported. Therefore, we analyzed mutant versus control mice using cell type specific markers. Compared to controls, the anterior aspect of the cochlear nucleus was altered in $W n t 1^{s w / s w}$ mice (Figures 10A-C). Specifically, the AVCN was shorter along the D-V axis and broader along the $\mathrm{M}-\mathrm{L}$ axis, which resulted in a subtle change in morphology (Figures 10B,C). We also observed the presence of large, ectopic Purkinje-like cells (PLC) in the anterior-dorsal aspects of the $W n t 1^{s w / s w}$ DCN (Figures 10D-F, arrow). In contrast, the gross morphology of the PVCN and posterior DCN were largely unaffected, although their were aberrant CALB-expressing neurons interspersed amongst CALR-expressing neurons in the
DCN (Figures 10G-K). We also compared the inferior colliculus (schematic shown in Figure 11A) of $W n t 1^{+/+}$and $W n t 1^{s w / s w}$ mice by immunolabeling for PV and CALB (Figures 11B-D). PV is a broad marker of the three inferior colliculus subdivisions, including the entire central nucleus (ICC) and aspects of the surrounding dorsal (DC) and lateral (LC) cortices (Coleman et al., 1992; Vater and Braun, 1994; Lohmann and Friauf, 1996; Sharma et al., 2009). CALB is present in the cortices surrounding the ICC, as well as the periaqueductal gray (PAG), located ventromedially (Coleman et al., 1992; Vater and Braun, 1994; Paxinos, 1999; Sharma et al., 2009). In the wild type auditory midbrain there was clear delineation between PV and CALB positive neurons (Figure 11B). Although much of the staining was diffuse due to PV and CALB positive afferents, the cell bodies of PV+ and CALB + neurons were readily identified in $W n t 1^{+/+}$ mice (Figure 11B, arrows and arrowheads, respectively). In $W n t 1^{s w / s w}$ mice, which exhibited a mild phenotype, the medial component of the PAG was distorted (Figure 10C, PAG $^{*}$ ). In addition, the commissural connections between colliculi of each side were not easily distinguished (compare bracketed areas in Figure 11B vs. Figure 11C). However, both PV and CALBexpressing neurons were abundant and generally positioned 


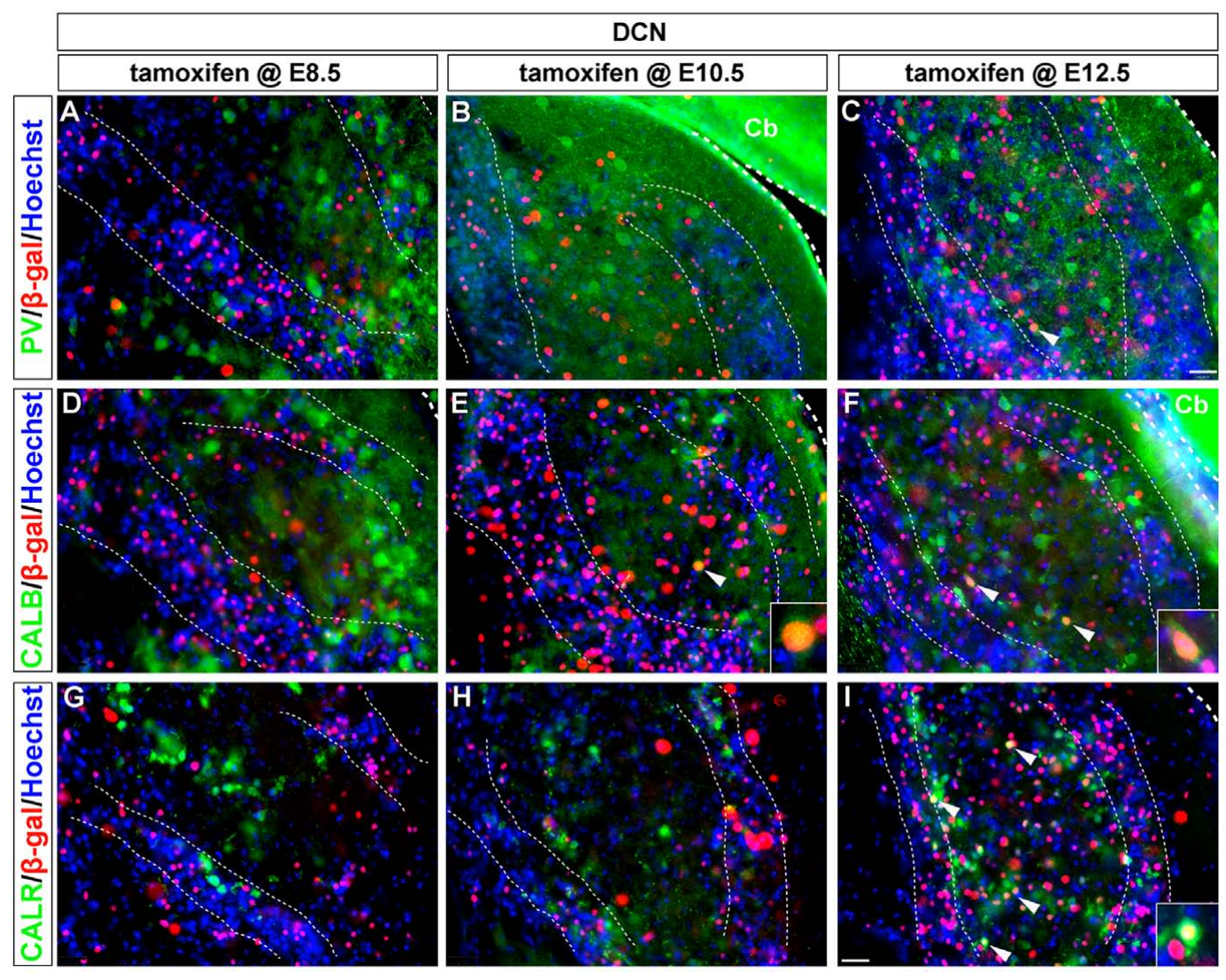

FIGURE 4 | CALB and CALR-expressing DCN neurons are sequentially derived from Wnt1-expressing progenitors. Wnt1-derived neurons were identified by nuclear $\beta$-gal immunocytochemistry (red) and with biochemical markers (green). (A-C) PV-expressing DCN neurons were not derived from progenitors which expressed Wnt1 between E8.5 and E12.5. (D-F) Progenitors expressing Wnt1 between E10.5 and E12.5 gave rise to CALB positive neurons only when marked at E10.5 and E12.5. (arrowheads, insets). (G-I) CALR positive DCN neurons were derived from Wnt1-expressing progenitors only when marked at E12.5 (see arrowheads, inset, I). Broad dashed lines indicate the separation of DCN from cerebellum (Cb), while fine dashed lines depict the granule cell layers. Scale bar in (I) indicates $32 \mu \mathrm{m}$.

in proper anatomical locations (Figure 11C, arrows and arrowheads, respectively). We observed $W n t 1^{s w / s w}$ mutants with a loss of bilateral symmetry, the presence of ectopic cell types, and aberrant A-P patterning in the midbrain, which we refer to as a severe phenotype (Figure 11D). Notably, the presumptive inferior colliculi were displaced along the $\mathrm{D}-\mathrm{V}$ and $\mathrm{A}-\mathrm{P}$ axes, and contained fewer PV-expressing cell bodies than controls (Figure 11D, IC*, right side in this example). CALB-expressing neurons were easily identified (Figure 11D, arrowheads), but there was no clear delineation of the PAG, DC, or LC. The presumptive superior colliculus (SC*) in $W n t 1^{s w / s w}$ mutants, based on morphology and laminated expression of CALB, was found in plane with the presumptive ICC (Figure 11D). Ectopic cerebellar Purkinje cell types as determined by morphology and co-expression of PV/CALB were found in clusters throughout the midbrain (Figure 11D, open arrowheads). In addition to morphological and cellular differences, afferents coursing through the ICC delineated by silver staining in $W n t 1^{s w / s w}$ mice were disorganized as they passed through the brachium en route to higher order auditory centers (Figures 12A,B vs. Figures $12 \mathrm{C}, \mathbf{D})$. These results confirm that $W n t 1$ is required for the normal specification of auditory midbrain neurons as well as the proper organization of commissural connections and afferents of the inferior colliculus.

\section{DISCUSSION}

We used Wnt1-Venus embryos to validate that Wnt1 expression dynamically changes at early stages and rapidly becomes fixed following neurulation. We then used GIFM to quantify the contribution of Wnt1-expressing progenitors to the auditory hindbrain and midbrain. We also took advantage of GIFM to assess two distinct models of how Wnt1-expressing progenitors contribute to biochemically distinct neurons of the auditory hindbrain and midbrain. Specifically, we tested whether auditory structures as well as unique cell types were derived entirely from an early pool of Wnt1-expressing progenitors (progressive lineage restriction model) or whether waves of Wnt1 expression (de novo expression model) are related to the competence of Wnt1-expressing progenitors that contribute to mature auditory structures. Interestingly, the de novo expression model 


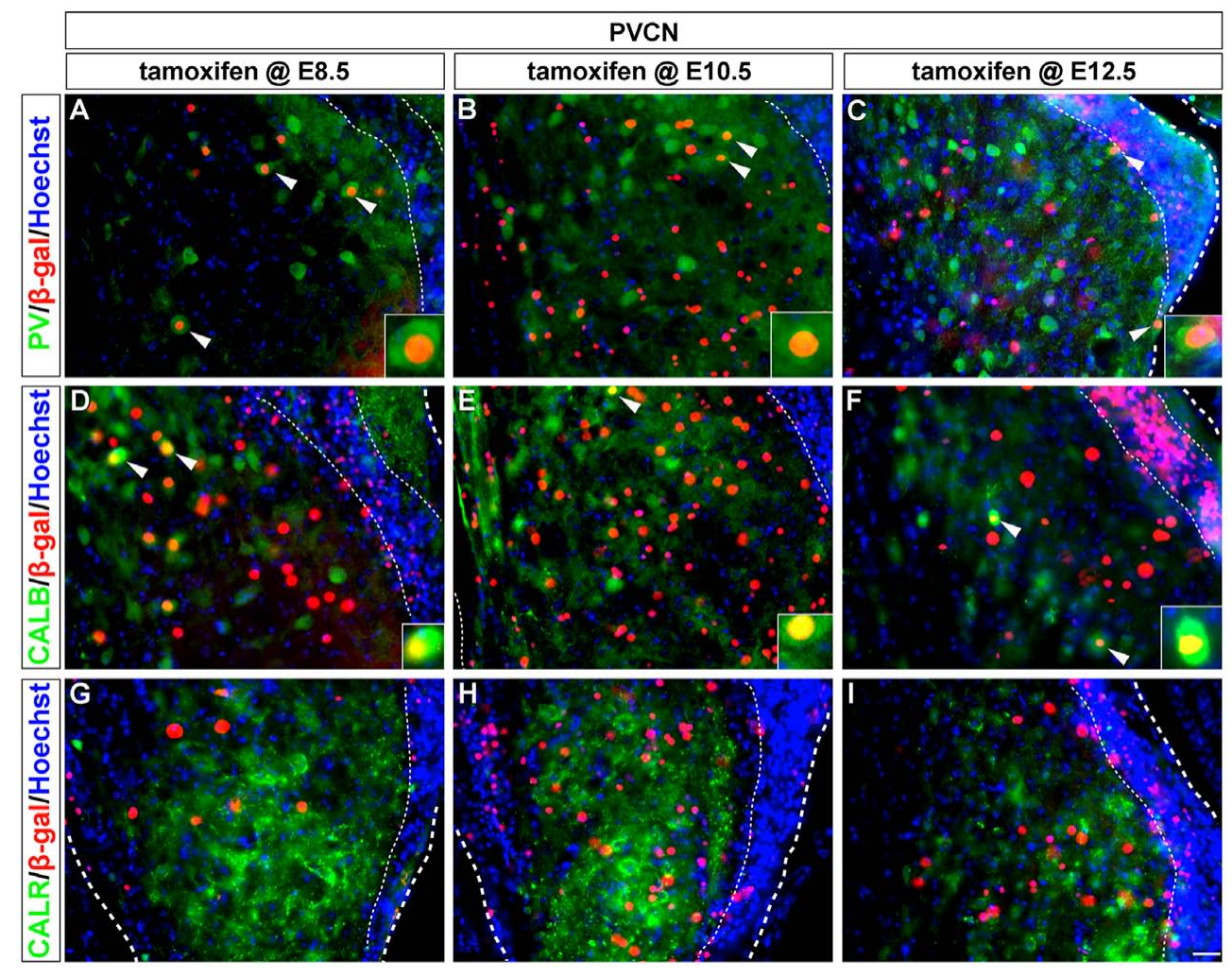

FIGURE 5 | PV and CALB-expressing PVCN neurons are derived from Wnt1-expressing progenitors. Wnt1-derived neurons were identified by nuclear $\beta$-gal immunocytochemistry (red) and biochemical markers were delineated in green. PV positive (A-C, arrowheads, insets) and CALB positive (D-F, arrowheads, insets) neurons of the PVCN were descendant from progenitors that expressed Wnt1 between E8.5 and E12.5. (G-I) CALR positive neurons were not derived from progenitors which expressed Wnt1 from E8.5 to E12.5. Broad dashed lines indicate lateral or medial edge of the PVCN; fine dashed lines depict the granule cell layer. Scale bar in (I) indicates $32 \mu \mathrm{m}$.

appropriately explains the contribution to the hindbrain. In contrast, a mixed model dominated by progressive lineage restriction best explains the contribution to the midbrain. Thus, both mechanistic models are employed in the development of Wnt1-derived auditory structures. Finally, we examined the morphology and cell types of the cochlear nucleus and inferior colliculus in $W n t 1^{s w / s w}$ mice and show minor deficits in the cochlear nucleus, but more severe perturbations in the inferior colliculus, which suggests that perturbing the lineage restriction model has a more dramatic impact on auditory brain structures.

\section{Wnt1 Lineage Contribution to the Auditory Hindbrain}

We utilized GIFM to characterize the profile and distribution of neurons derived from the Wnt1 lineage and showed that the Wnt1 lineage contributes to the cochlear nucleus in complex waves of de novo Wnt1 expression. This model is nicely illustrated by assessing the contribution to the DCN. Notably, if the DCN was derived entirely from progenitors expressing Wnt1 early, we would have expected a contribution to all cell types including granule cells and magnocellular neurons. However, we observed that the MNS (granule neurons) is derived from Wnt1-expressing progenitors throughout the span of Wnt1 expression (E8.5-E12.5), but the large projecting neurons of the magnocellular core are generated after E10.5. Notably, the competence state of progenitors to give rise to biochemically distinct cell types does not diminish as development proceeds. Therefore, de novo expression of $W n t 1$ in auditory progenitors occurs within the LRL after E10.5 to generate CALB+ and CALR+ neurons of the DCN. We quantified the contribution of Wnt1-expressing progenitors to cochlear nuclei over time and show that de novo peaks of Wnt1 expression occurred. These results show that despite broad expression of Wnt1 throughout the LRL germinal zone, progenitors exist that do not express Wnt1 early, but turn it on at later developmental stages.

\section{Wnt1 Lineage Contribution to the Auditory Midbrain}

The inferior colliculus of the midbrain is a complex structure and harbors neurons that express periodicity proteins, which regulate a circuidian clock (Park et al., 2016). In addition, the inferior colliculus is innervated by dopamine neurons of the subparafascicular thalamic nucleus (Nevue et al., 2016). Our fate mapping results clarify how neurons are established and contribute to the mature auditory midbrain. Specifically, 

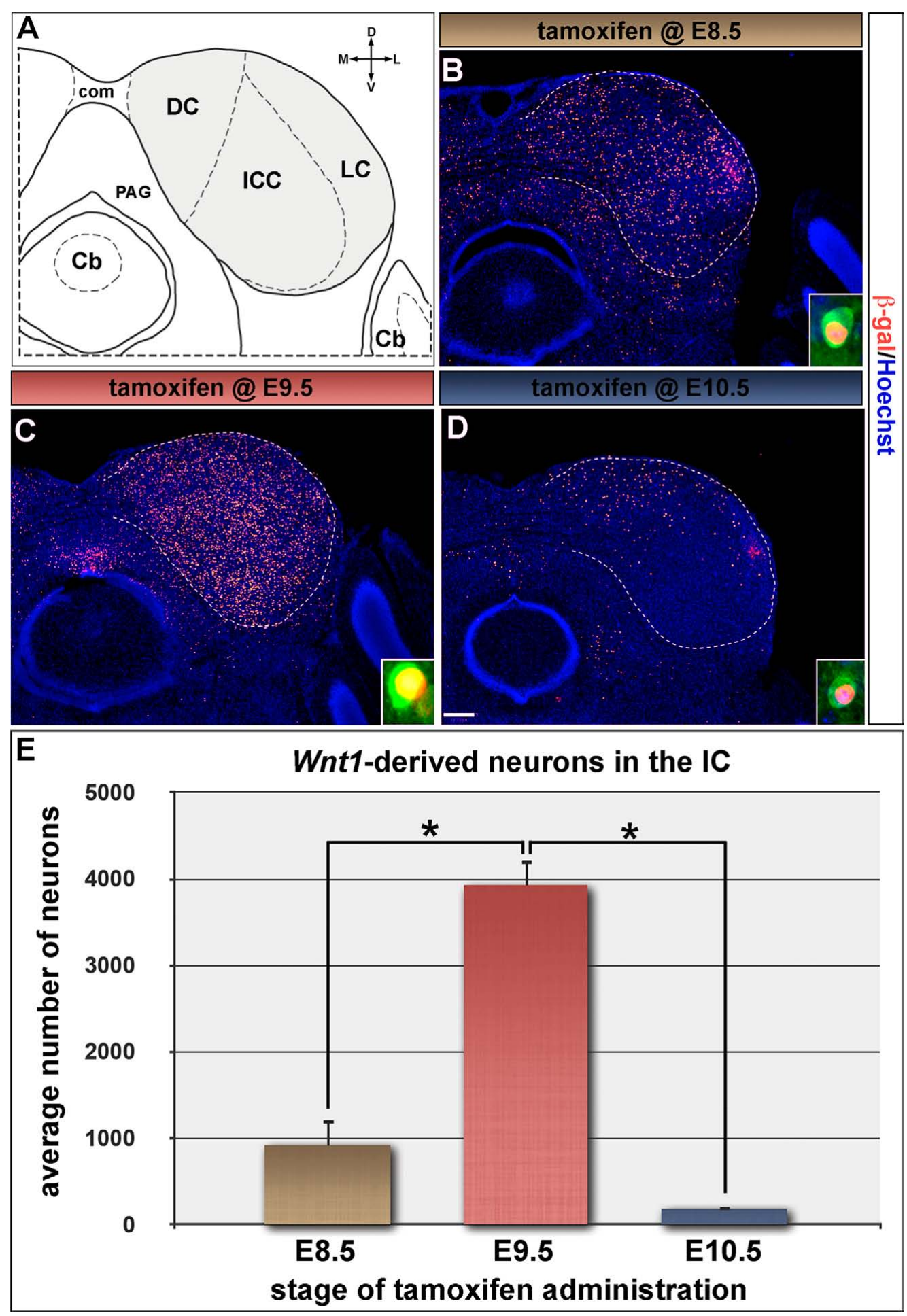

FIGURE 6 | The inferior colliculus is established by progenitors expressing Wnt1 between E8.5 and E10.5. (A) Schematic representation of the auditory midbrain (hemicoronal view is shown). The inferior colliculus (IC) is shaded gray to show the areas counted in (E), which are partitioned by dashed lines. (B-D) Wnt1-derived neurons were identified by nuclear $\beta$-gal immunocytochemistry (red). (B,E) Wnt1-expressing progenitors marked at E8.5 gave rise to $919.3 \pm 307.5$ Wnt1-derived IC neurons/section. (C,E) The pool of IC neurons is largely established by Wnt1 expressing descendants marked at E9.5 (3923.8 \pm 722.7 Wnt1-derived IC

neurons/section). (D,E) Wnt1-expressing progenitors at E10.5 contributed sparsely to IC neurons (181.7 \pm 132.8 Wnt1-derived IC neurons/section), which largely populated the dorsomedial IC. Insets in (B-D) depict representative PV positive neurons of the ICC derived from Wnt1-expressing progenitors. Asterisks denote average numbers of Wnt1-derived neurons which were significantly different ( $p<0.001)$. Scale bar: $260 \mu \mathrm{m}$ (B-D). 


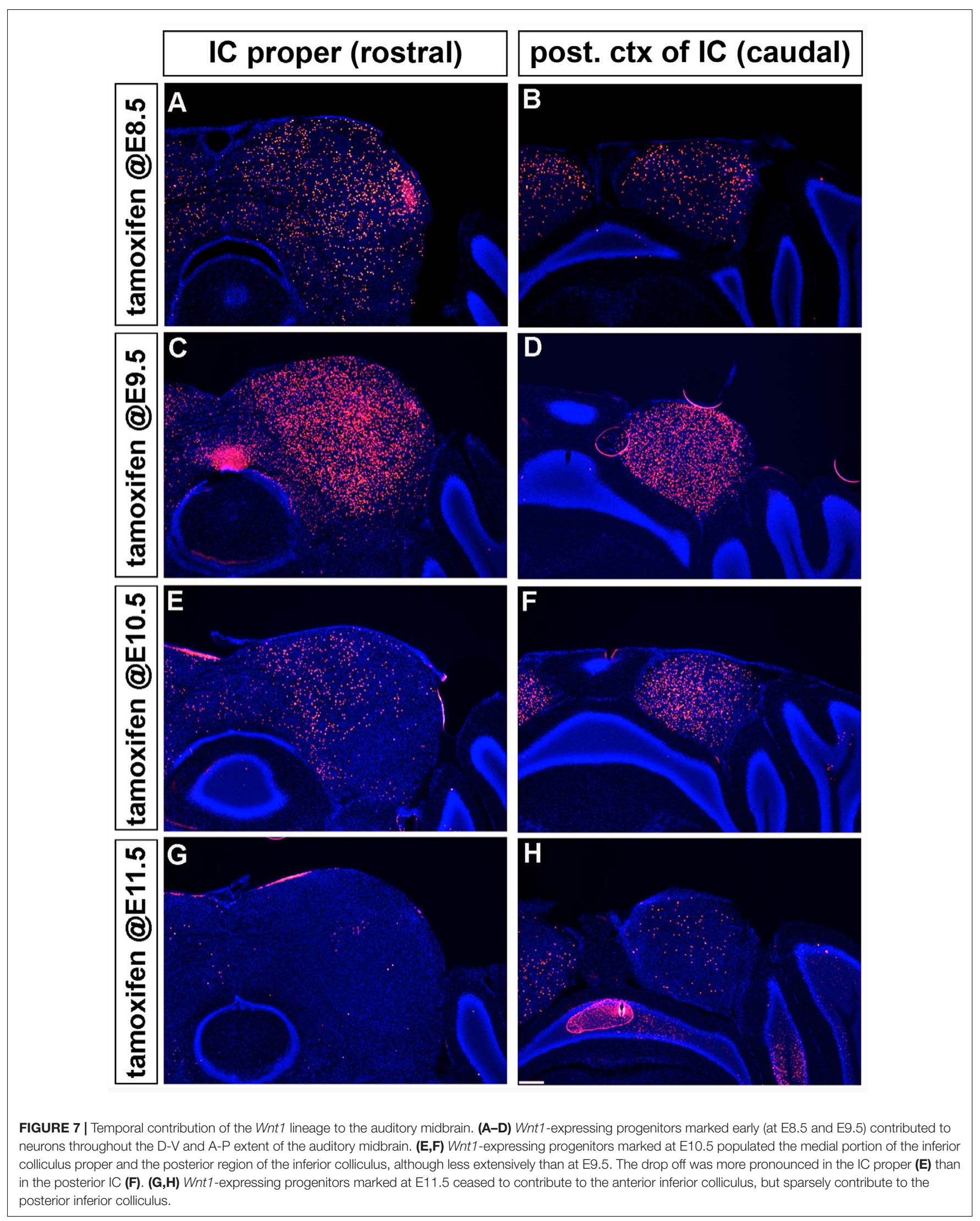




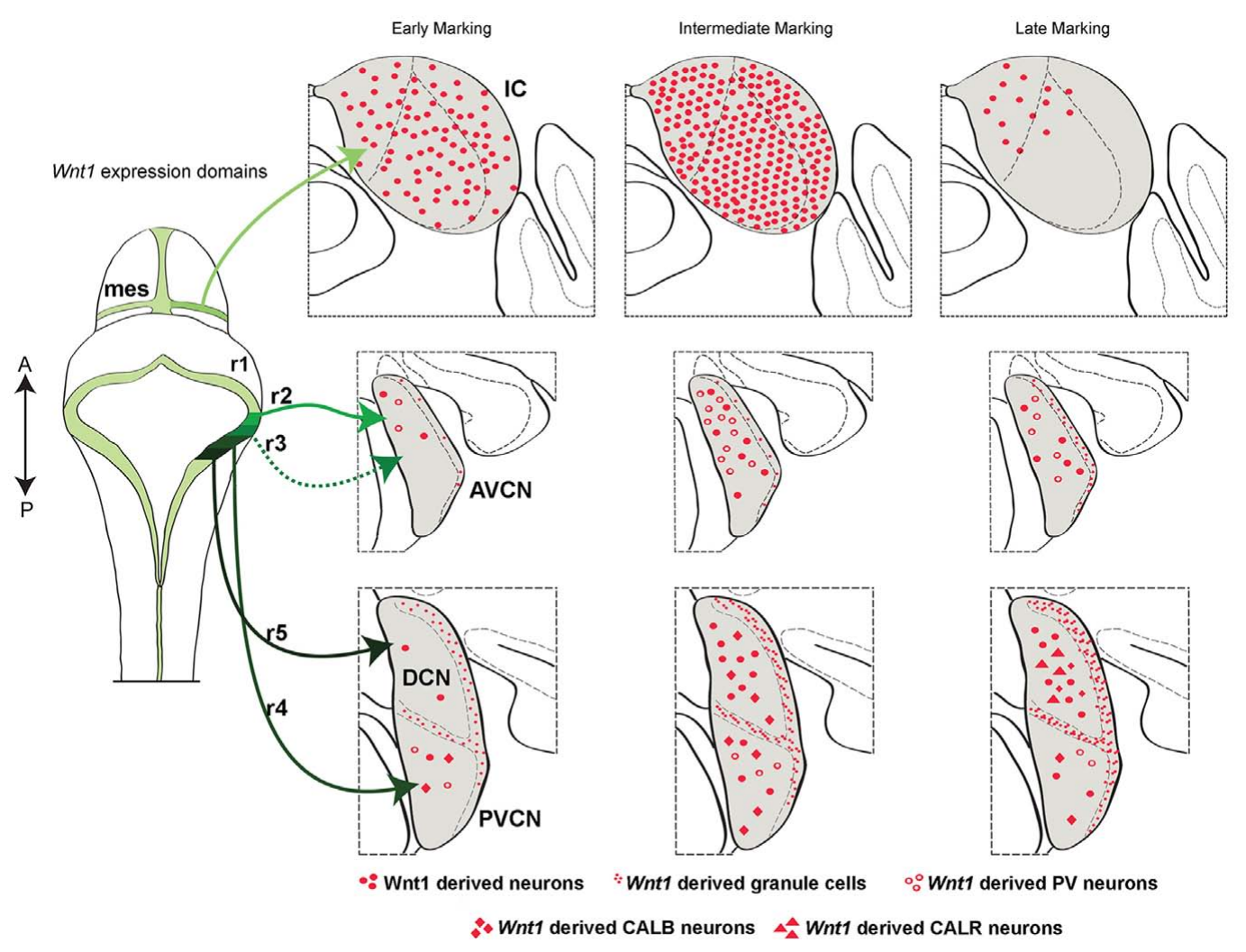

FIGURE 8 | Summary schematic of the temporal allocation of Wht1-expressing progenitors to mature midbrain and hindbrain auditory domains. A schematic of the dorsal view of the mouse mid-hindbrain during embryogenesis shows the Wnt1 expression domains (green-black) and the contributions from the mesencephalon (mes) and rhombomeres ( $r$ ) 2-5 to the mature inferior colliculus (IC) and cochlear nuclei. The IC was derived from Wnt1-expressing progenitors in the dorsal mes. Note: specific rhombomere contributions are based on Farago et al. (2006). Early, intermediate, and late marking is correlated to specific structures as indicted. Light green lines show the Wnt1 lineage contribution to the auditory midbrain: Early (E8.5) marking of Wnt1-expressing progenitors in the mes had a diffuse contribution to the IC. Intermediate (E9.5) marking produced the peak contribution to the IC. Late (E10.5) marking conferred sparse contribution to dorsomedial aspects of the inferior colliculus. Intermediate green lines indicate Wnt1 lineage contribution to auditory hindbrain cochlear nuclei: The AVCN is derived from Wnt1-expressing progenitors positioned in $\mathrm{r} 2$ and $\mathrm{r} 3$. Early marking (E8.5) of Wnt1-expressing progenitors resulted in sparse contribution to PV+ neurons (open circles) in the dorsal tier of the AVCN. Intermediate marking (E10.5) revealed greater contribution to PV+ neurons, while late (E12.5) marking resulted in a decrease of Wnt1-derived neurons in the AVCN. Black lines show the DCN is derived from Wnt1-expressing progenitors located in r5. Early marking (E8.5) of Wnt1-expressing progenitors revealed a primary contribution to granule cells of the MNS (small red dots). Magnocellular neurons of the DCN were derived from progenitors expressing Wnt1 at an intermediate stage (E10.5), including CALB-expressing neurons (red diamonds). CALR-expressing neurons (red triangles) were derived from late (E12.5) Wnt1 expression. Dark green line shows that the PVCN is derived from Wnt1-expressing progenitors of $r 4$. The distribution of neurons derived from Wnt1-expressing progenitors resembles that of the AVCN, though PV+ and CALB+ neurons were derived from each stage examined.

we show that the inferior colliculus is not entirely derived from progenitors that express Wnt1 early, which is consistent with the presence of both Wnt1-expressing and non-expressing progenitors that are intermingled within the mesencephalon at E8.5 (Figure 1). Thus, Wnt1-expressing progenitors marked at E8.5 results in a relatively diffuse contribution through the extent of the inferior colliculus (Figure 8). Wnt1 expression in the mesencephalon dynamically changed, coinciding with the closure of the anterior neural fold (at E9.5). Notably, the peak contribution to inferior colliculus neurons arose from Wnt1-expressing progenitors marked at E9.5. These findings imply that de novo Wnt1 expression occurred in the dorsal mesencephalon over the span of $24 \mathrm{~h}$. Subsequently, the contribution of Wnt1-expressing progenitors to the inferior colliculus rapidly decreased (with marking at E10.5). A model of progressive lineage restriction best describes how progenitors contribute to the inferior colliculus following neural tube closure. Collectively, our results show that the auditory midbrain is established by a mixed model of Wnt1 lineage-contribution in which an early wave of de novo expression is replaced by a progenitor population that is progressively restricted over time.

\section{GIFM of the Wnt1 Lineage versus Birth Dating in Auditory Structures}

In the current study we used a Swiss Webster (mouse) background for lineage mapping. However, studies using ${ }^{3} \mathrm{H}$ thymidine labeling for birth dating of neurons were typically conducted in rat (Altman and Bayer, 1981). These classic studies 


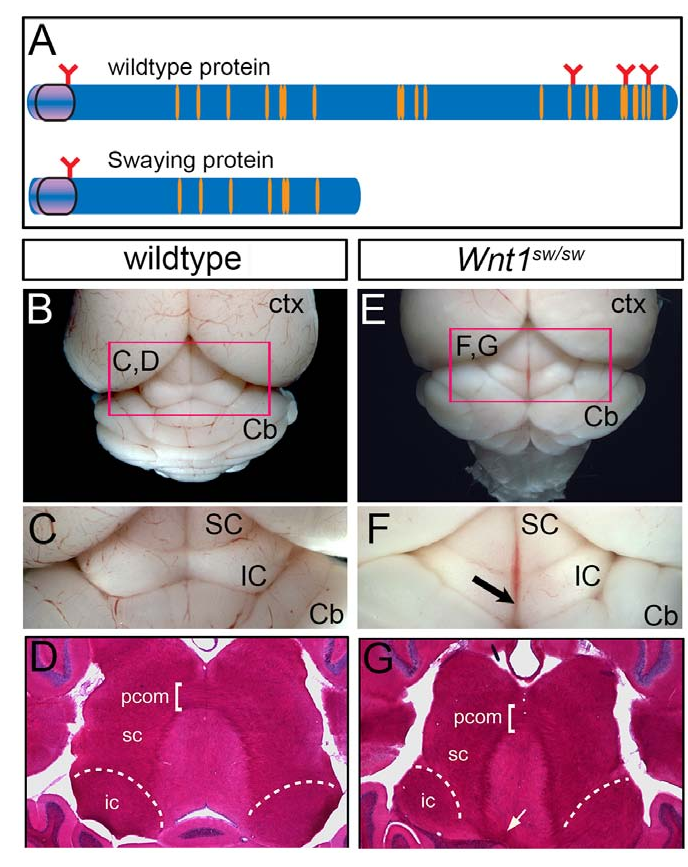

FIGURE 9 | Whole mount view and horizontal sections showing adult brain structures of wild type and $W n t 1^{s w / s w}$ mice. (A) Schematic of wild type WNT1 and Swaying mutant proteins; orange indicates cysteine residues, red indicates glycosylation sites. (B) Wild type whole-mount photomicrograph showing the caudal aspect of the cerebral cortex (Ctx) and cerebellum (Cb) at low magnification. The dorsal midbrain is delineated by the pink marque. (C) The superior colliculus (SC) and inferior colliculus (IC) of a representative wild-type mouse is shown at higher magnification. (D) A horizontal section stained with hematoxylin and eosin (H\&E) shows the dorsal midbrain cytoarchitecture of a control wild-type mouse including the posterior commissure (pcom). (E) Whole-mount photomicrograph of a representative Wnt $1^{\text {sw/sw }}$ mouse showing the caudal aspect of the cerebral cortex (Ctx) and the cerebellum $(\mathrm{Cb})$ with midline/vermis defect at low magnification. The dorsal midbrain is perturbed as compared to wild-type mouse. (F) In particular, the IC is misshaped and has a lack of continuity at the midline (arrow). (G) A horizontal section of a Wnt1 ${ }^{\mathrm{SW} / \mathrm{SW}}$ mouse stained with H\&E shows aberrant fiber tracts emanating from adjacent to the inferior colliculus (white arrow).

have been the gold standard of birth dating. Thus, we converted rat to mouse embryonic stages of development (Supplementary Table 1) to contrast our GIFM with birth dating studies. Using this approach for comparison indicates there is contribution of the Wnt1 lineage at E8.5mouse (E9.6rat) with the peak of contribution along the rostral-caudal axis occurring at E9.5mouse (E10.8 rat ) with a significant decrease at E10.5 mouse (E12.0 rat). The lateral aspect of the inferior colliculus has a peak of birth dating between E16 and E17 rat which is estimated to be postE12.5 mouse. However, at E12.5 mouse the Wnt1-lineage no longer contributes the inferior colliculus. Similarly, the medial aspect of the inferior colliculus has a peak of birth dating between E17 and E19 rat which is estimated to be E15.5-E16.6 mouse. Notably, this time period is also later than Wnt1 expression and lineage contribution in the inferior colliculus. Finally, a similar trend is observed in the DCN of the hindbrain: peak birth dating of the DCN is E14-E16rat (E12-E14 mouse) (Altman and Bayer, 1980). Thus, birth dating occurs subsequent to Wnt1 lineage allocation, which happens between E10.5-E12.5 in mouse. In summary, Wnt1 is extinguished prior to terminal proliferation and suggests that genetic lineage is established in the inferior colliculus and DCN of the hindbrain earlier than what might be suggested by birth dating studies. Interestingly, a similar observation was found when using GIFM to determine Gli1 lineage allocation during dopamine neuron development (Hayes et al., 2011). However, this is not a universal theme as Wnt1 lineage allocation occurs concomitantly with birth dating in the cerebellum and pre-cerebellar system (Hagan and Zervas, 2012). More broadly and in the context of the current study, these comparisons suggest that the Wnt1 lineage is established prior to birth dating in the auditory midbrain and hindbrain.

\section{Wnt1 Is Required for the Development of Central Auditory Structures}

Notably, Wnt $1^{s w / s w}$ mice have a truncated and broadened AVCN and the presence of ectopic PLCs in the anterior DCN (Figure 10). The presence of these cells, which have been referred to as displaced Purkinje cells or PLCs, has been observed in the brainstem of wild type rats, including in the cochlear nucleus (Hurd and Feldman, 1994; Koszeghy et al., 2009). In comparison, the location of PLCs within the DCN of $W n t 1^{s w / s w}$ mice suggests that the expression of Wnt1 normally prevents their presence or specification within this region in mice. Wnt1 is also required for the development of the auditory midbrain. In both mildly and severely affected $W n t 1^{s w / s w}$ mice, the central nucleus and external cortex of the inferior colliculus were present. However, the auditory midbrain in severely affected mice was aberrantly patterned. Severe $W n t 1^{s w / s w}$ mutants had ectopic anterior midbrain and misplaced cerebellar tissues surrounding the presumptive inferior colliculus. In one example, half of the auditory midbrain was largely void of PV + cell bodies, and commissural connections between each Inferior colliculi could not be detected. In addition, axons of the IC were greatly disorganized. It is known that the loss of $W n t 1$ perturbs the formation of the isthmus organizer required for patterning the adjacent midbrain and cerebellum (Adams et al., 2000; Matsunaga et al., 2002; Ellisor et al., 2012), yet how this perturbation affected the development of the inferior colliculus and its subdivisions had not been determined. While it is likely that much of the patterning deficits we observe are secondary to the disruption of the isthmus organizer, Wnt1 is apparently also required for the proper specification of auditory structures and their connections.

One prominent deficit common to both mildly and severely affected $W n t 1^{s w / s w}$ mice is the loss or displacement of commissural connections between the colliculi. Moreover, the axonal output of the inferior colliculus was greatly disorganized in mildly affected mice. Late Wnt1 expression may therefore be instructive in establishing inter-collicular projections or to promote the outgrowth of anterior projections en route to the medial geniculate body in the thalamus. Lastly, the 

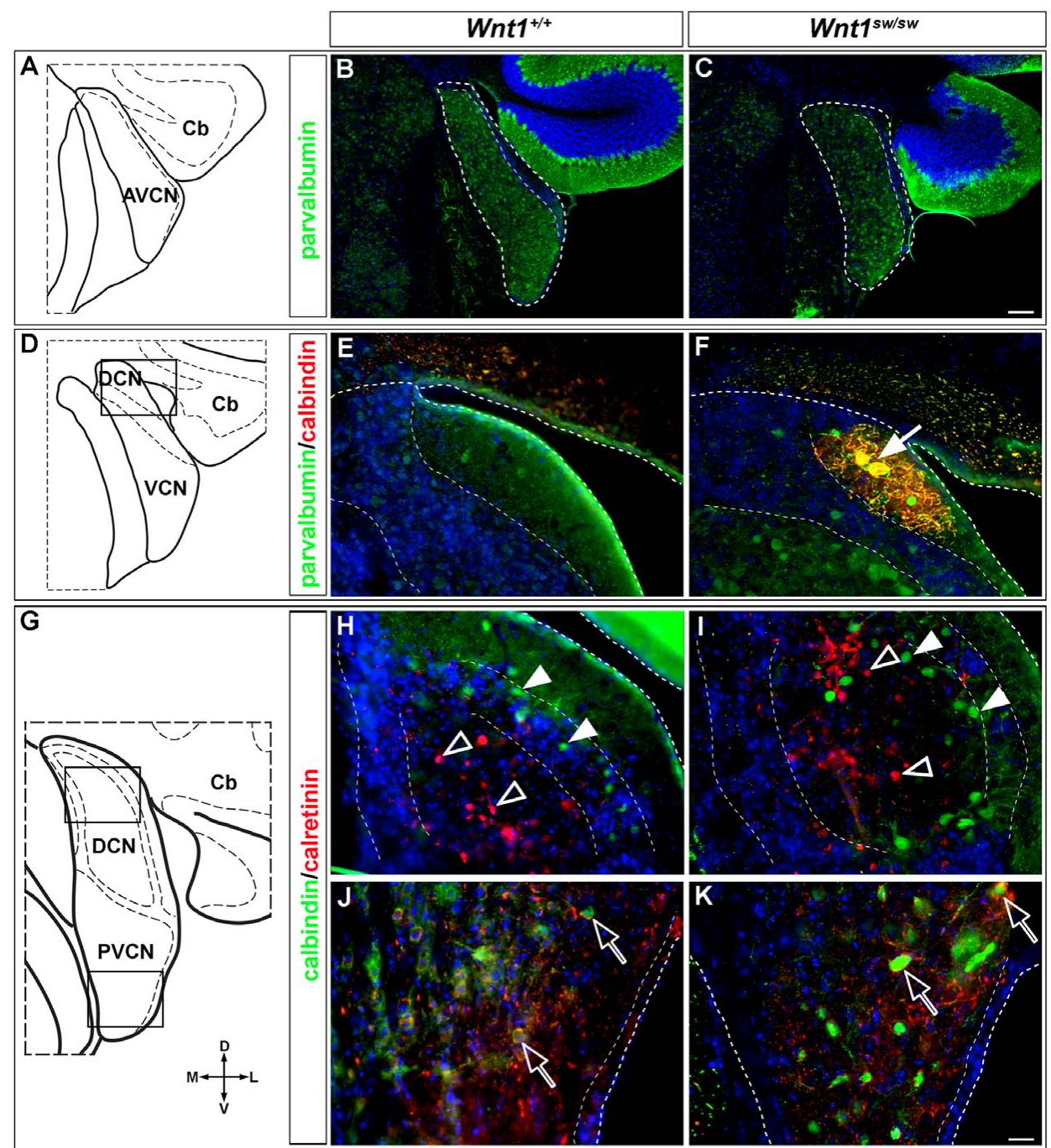

FIGURE 10 | Altered morphology and ectopic cell types in the cochlear nucleus of Wnt $1^{\text {sW/sW }}$ mice. (A) Schematic representation of the AVCN. (B) Normal morphology observed in control AVCN. (C) Stereotypical and broadened morphology observed in the AVCN of Wnt1sw/sw mice. (B,C) PV-expressing neurons of the AVCN were prevalent in controls and mutants. (D) Schematic view of the DCN/VCN in sectioning planes and boxed areas analyzed in (E,F), depicting the transition between AVCN and PVCN (denoted VCN) and the most anterior aspects of the DCN. While the general morphology of mutant posterior cochlear nuclei remained intact, an ectopic cell type characteristic of Purkinje-like cells (PLCs, white arrow) based on morphology and co-expression of PV/CALB) was commonly observed (F) in mutants versus controls (E). (G) Schematic view delineating the boxed areas of the DCN and PVCN analyzed in (H-K). (H,I) The general morphology of the DCN was largely normal in controls versus mutants. CALB (solid arrowheads) and CALR (open arrowheads) expressing neurons were generally unaffected although some CALB+ neurons were inappropriately localized to the core. The morphology and cell type specification appeared to be unaffected in the mutant PVCN. Open arrows highlight CALB+ neurons surrounded by CALR+ positive processes. Scale bars: $130 \mu \mathrm{m}$ (B,C); $32 \mu \mathrm{m}$ (E,F,H,I,J,K).

presence of a late WNT1 signal in the mesencephalon may serve as an attractant or remodeling cue for ascending input from developing hindbrain auditory centers. Projections from the cochlear nucleus reach the midbrain as early as E15 in rat, which is on par with late Wnt1 expression in the dorsal mesencephalon (note: E15 in rat roughly corresponds to E13 in mouse (Clancy et al., 2001 and Supplementary Table 1). This suggests a mechanism of molecular matching where auditory neurons of temporally defined lineages may be functionally connected by axonal projections.

\section{Wnt1 Lineage Contribution to Auditory Structures versus Wnt1 Mutant Phenotypes}

Although we demonstrated that Wnt1 is required for proper development of the hindbrain cochlear nucleus and the midbrain inferior colliculus using mice with a perturbation in Wnt1, it may appear that there is discordance between our fate mapping results and the $W n t 1^{s w / s w}$ phenotype. However, subtle differences between fate mapping results and the $W n t 1^{s w / s w}$ phenotype could be due to the fact that the population of Wnt1-expressing cells 

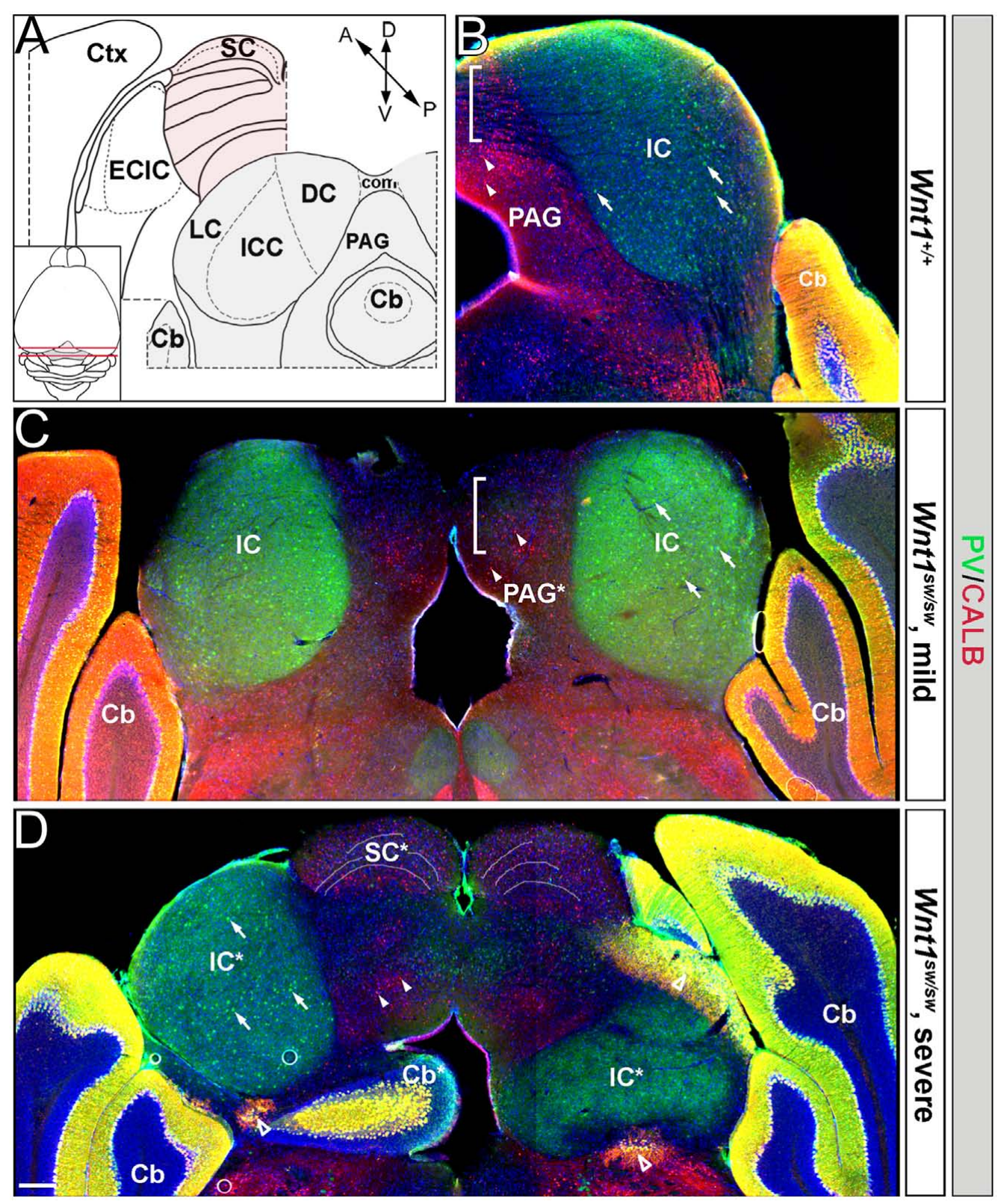

FIGURE 11 | Patterning deficits of the auditory midbrain of $W n t 1^{\text {sw/sw }}$ mice. (A) Schematic representations of wild type hemi-coronal planes of section through the anterior and posterior dorsal midbrain. The posterior midbrain is analyzed in (B-D). Inset: schematic of a dorsal whole mount brain depicts the anterior (pink shading) and posterior (gray shading) midbrain. The anterior diagram shows the superior colliculus (SC, highlighted in pink) and the external cortex of the inferior colliculus (ECIC, highlighted in gray). Laterally, the cortex (Ctx) surrounds the Mb in this plane. The posterior plane delineates each subdivision of the inferior colliculus proper: central nucleus (ICC), dorsal cortex (DC), and lateral cortex (LC). The periaqueductal grey (PAG) borders the inferior colliculus ventromedially. The cerebellum is denoted (Cb). (B) Hemi-coronal wild type section representative of the posterior plane shows normal staining of PV (green) and CALB (red). PV is broadly seen throughout the inferior colliculus (IC) while CALB is largely confined to the PAG. Examples of PV and CALB positive neurons are noted with arrows and arrowheads, respectively. Commissural connections between the colliculi are indicated by the bracketed area and by (com) in (A). Phenotypes varied from mild (C) to severe (D) in $W n t 1^{s w / s w}$ mice. (C) $W n t 1^{s W / s w}$ mice with a mild phenotype exhibited minor changes in bilateral symmetry while the general morphology of each IC was maintained. Although the PAG was malformed (denoted by PAG*) both PV and CALB neurons were specified. Commissural connections seen as black striations in wild-type mice (B) were absent in the bracketed domain (D). Wht $1^{\mathrm{sW} / \mathrm{sw}}$ mice with a severe phenotype displayed a loss of bilateral symmetry between each IC (as determined by PV expression and denoted as IC*) The severe phenotype also had ectopic cerebellar tissue and cell types within the midbrain. Finally, there was the presence of presumptive SC tissue that was positioned anteriorly in controls (see illustration in A). The left IC* in (D) was established with apparently normal morphology and contained PV-expressing neurons, while the right IC* was severely perturbed and contained few PV-expressing neurons. Ectopic or malformed $\mathrm{Cb}$ tissue was present within the $\mathrm{Mb}\left(\mathrm{Cb}^{*}\right)$, which contained ectopic Purkinje cells that expressed both PV and CALB (open arrowheads). Presumptive anterior tissue (SC*) was inappropriately positioned and was observed in the same plane as the IC (compare $\mathbf{B}$ and $\mathbf{D}$ ), which was evident by the dorsal laminated morphology and expression of CALB. Scale bar: $260 \mu \mathrm{m}$ (B-D). 

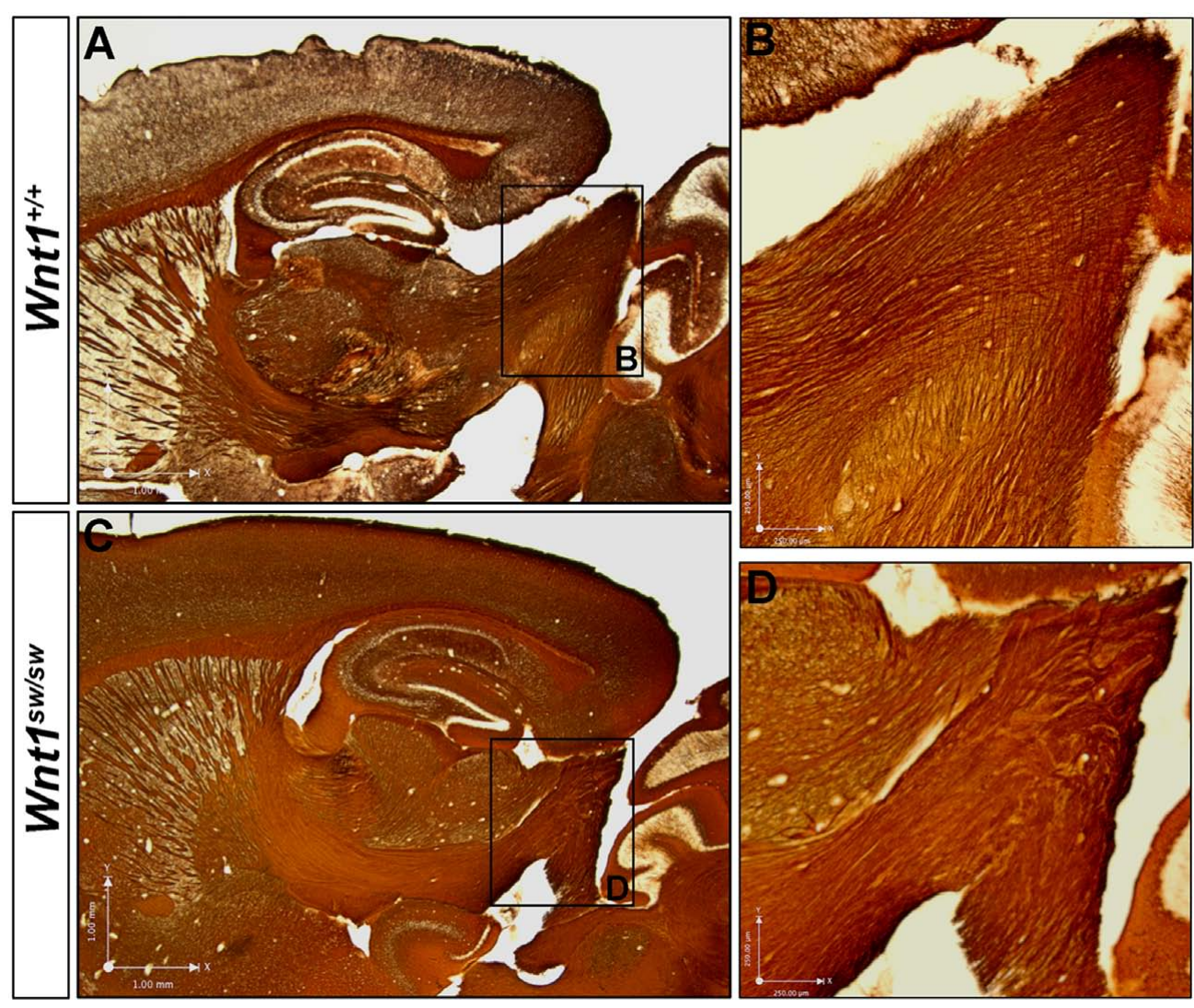

FIGURE 12 | Wht1 is required for normal axonal organization in the brachium of the inferior colliculus. Myelin-based silver staining allowed for the visualization of axons leaving the inferior colliculus en route to higher order auditory centers. (A,B) Wild type axons apparently exiting the auditory midbrain formed organized, parallel fibers. (C,D) Wnt T $^{s w / s w}$ mice displayed clumped, disoriented axonal bundles. See Figure 9 for morphological changes in the inferior colliculus and ectopic white matter fibers in horizontal orientation.

(marked by GIFM) and the population of cells that respond to Wnt1 signaling are not identical and might only partially overlap. Additionally, GIFM results in a mosaic tapestry of cells marked within a distinct temporal window, while the $W n t 1^{s w / s w}$ line is the result of a germ line mutation, which may contribute to differences. Finally, it cannot be fully excluded that Wnt1-CreER ${ }^{T}$ expression differs (slightly) from endogenous Wnt1 expression. However, it can be construed that divergent expression of Wnt1$\mathrm{CreER}^{T}$ and $W n t 1$ is unlikely since our previously published analysis of the initial marked populations using this line did not provide evidence that the two expression patterns differ from each other (Ellisor et al., 2009; Hagan and Zervas, 2012).

\section{Potential Roles of Wnt1 in Auditory System Development}

The Wnt1 lineage which gives rise to the hindbrain cochlear nucleus is not progressively restricted over time. We show that waves of de novo Wnt1 expression are differentially correlated with the distribution and emergence of unique cell types, which suggests that Wnt1 may not play a primary role in promoting the proliferation of cochlear nucleus progenitors. Rather, our results suggest temporal roles for Wnt1 in specifying distinct cell types within the cochlear nucleus subdivisions. A notable finding is that Wnt1 contributes to functional classes of auditory hindbrain neurons. While we hypothesized that biochemically distinct neurons (expressing PV, CALB, or CALR) would be differentially derived based on the timing of Wnt1 expression, this was true only for CALB and CALR neurons of the DCN. Interestingly, the biochemical signature in each case correlates with neurons that form contralateral excitatory circuits (Fredrich et al., 2009). This could represent a mechanisms where lineage allocation and neural circuits are coupled. The Wnt1 lineage primarily established excitatory cell types such as PV-expressing neurons of the AVCN/PVCN. These cells are thought to be T-stellate/multipolar type I neurons that provide excitatory contralateral innervation of the inferior colliculus (Cant and Benson, 2003).

Outstanding questions are what are the downstream molecular targets of Wnt1 and how might they help decipher a pathway used in development? Through cumulative genetic fate mapping, Fujiyama demonstrated that progenitors that express the bHLH transcription factors Math1 and Ptf1a give rise to excitatory and inhibitory cell types of the cochlear nucleus, respectively. Math1 is contained within the dorsal tier of Wnt1 expression in the LRL (Landsberg et al., 2005), while Ptf1a is 
believed to be expressed just ventral to or slightly overlapping with the weakest ventral domain of $W n t 1$ expression (Yamada et al., 2007; Fujiyama et al., 2009). Differing levels of WNT1 may regulate the expression of these transcription factors, such that high levels promote Math1, while low levels promote Ptf1a. Of importance is determining whether these genes are regulated by canonical Wnt signaling, as demonstrated for neural crest lineages of the developing hindbrain (Lee et al., 2004). Regardless, our fate mapping studies provide a comprehensive assessment of the how temporal expression of $W n t 1$ is related to auditory lineage allocation and we provide functional evidence for the role of Wnt1 in the development of the auditory hindbrain and midbrain.

\section{ETHICS STATEMENT}

Mice were housed and handled in accordance with Brown University Institutional Animal Care and Use Committee

\section{REFERENCES}

Adams, K. A., Maida, J. M., Golden, J. A., and Riddle, R. D. (2000). The transcription factor Lmx1b maintains Wntl expression within the isthmic organizer. Development 127, 1857-1867.

Aitkin, L. M., and Phillips, S. C. (1984). Is the inferior colliculus an obligatory relay in the cat auditory system? Neurosci. Lett. 44, 259-264.

Altman, J., and Bayer, S. A. (1980). Development of the brain stem in the rat. Ill. Thymidine-radiographic study of the time of origin of neurons of the vestibular and auditory nuclei of the upper medulla. J. Comp. Neurol. 194, 877-904. doi: $10.1002 /$ cne. 901940410

Altman, J., and Bayer, S. A. (1981). Time of origin of neurons of the rat inferior colliculus and the relations between cytogenesis and tonotopic order in the auditory pathway. Exp. Brain Res. 42, 411-423. doi: 10.1007/BF00237506

Bally-Cuif, L., Cholley, B., and Wassef, M. (1995). Involvement of Wnt-1 in the formation of the mes/metencephalic boundary. Mech. Dev. 53, 23-34. doi: 10.1016/0925-4773(95)00421-1

Bredderman, P. J., and Wasserman, R. H. (1974). Chemical composition, affinity for calcium, and some related properties of the vitamin D dependent calciumbinding protein. Biochemistry 13, 1687-1694. doi: 10.1021/bi00705a021

Bronson, R. T., and Higgins, D. C. (1967). The swaying (sw) mutation causes a midline sagittal fissure. Mouse Genome 89:851.

Brown, A., Brown, S., Ellisor, D., Hagan, N., Normand, E., and Zervas, M. (2009). A practical approach to genetic inducible fate mapping: a visual guide to mark and track cells in vivo. J. Vis. Exp. 34:1687. doi: 10.3791/1687

Brown, A., Machan, J. T., Hayes, L., and Zervas, M. (2011). Molecular organization and timing of Wnt 1 expression define cohorts of midbrain dopamine neuron progenitors in vivo. J. Comp. Neurol. 519, 2978-3000. doi: 10.1002/cne.22710

Caicedo, A., d'Aldin, C., Puel, J. L., and Eybalin, M. (1996). Distribution of calciumbinding protein immunoreactivities in the guinea pig auditory brainstem. Anat. Embryol. 194, 465-487. doi: 10.1007/BF00185994

Cant, N. B., and Benson, C. G. (2003). Parallel auditory pathways: projection patterns of the different neuronal populations in the dorsal and ventral cochlear nuclei. Brain Res. Bull. 60, 457-474. doi: 10.1016/S0361-9230(03)00050-9

Celio, M. R. (1990). Calbindin D-28k and parvalbumin in the rat nervous system. Neuroscience 35, 375-475. doi: 10.1016/0306-4522(90)90091-H

Ciani, L., and Salinas, P. C. (2005). WNTs in the vertebrate nervous system: from patterning to neuronal connectivity. Nat. Rev. Neurosci. 6, 351-362. doi: $10.1038 / \mathrm{nrn} 1665$

Clancy, B., Darlington, R. B., and Finlay, B. L. (2001). Translating developmental time across mammalian species. Neuroscience 105, 7-17. doi: 10.1016/S03064522(01)00171-3
(IACUC) guidelines (Genetic Approaches Using Mus Musculus as a Model Organism to Understand Mechanisms Underpinning Neurodevelopment and Neurological Disorders in Vivo", IACUC \#1209030).

\section{AUTHOR CONTRIBUTIONS}

The original experimental approach was designed by SB and MZ. Experiments were conducted by SB. The manuscript was written and edited by $S B$ and MZ. Research presented here fulfilled in part the Ph.D. thesis requirement for SB.

\section{SUPPLEMENTARY MATERIAL}

The Supplementary Material for this article can be found online at: http://journal.frontiersin.org/article/10.3389/fnana. 2017.00067/full\#supplementary-material

Coleman, J. R., McDonald, A. J., Pinek, B., and Zrull, M. C. (1992). The inferior colliculus: calbindin and parvalbumin immunoreactivity in neural grafts. Exp. Neurol. 115, 142-145. doi: 10.1016/0014-4886(92)90238-L

Di Bonito, M., and Studer, M. (2017). Cellular and molecular underpinnings of neuronal assembly in the central auditory system during mouse development. Front. Neural Circuits 11:18. doi: 10.3389/fncir.2017.00018

Dingle, Y. L., Xiong, K. B., Machan, J. T., Seymour, K. A., Ellisor, D., Hoffman-Kim, D., et al. (2016). Quantitative analysis of dopamine neuron subtypes generated from mouse embryonic stem cells. bioRxiv doi: 10.1101/09 3419

Ellisor, D., Koveal, D., Hagan, N., Brown, A., and Zervas, M. (2009). Comparative analysis of conditional reporter alleles in the developing embryo and embryonic nervous system. Gene Expr. Patterns 9, 475-489. doi: 10.1016/j.gep.2009. 07.007

Ellisor, D., Rieser, C., Voelcker, B., Machan, J. T., and Zervas, M. (2012). Genetic dissection of midbrain dopamine neuron development in vivo. Dev. Biol. 372, 249-262. doi: 10.1016/j.ydbio.2012.09.019

Ellisor, D., and Zervas, M. (2010). Tamoxifen dose response and conditional cell marking: Is there control? Mol. Cell. Neurosci. 45, 132-138. doi: 10.1016/j.mcn. 2010.06.004

Farago, A. F., Awatramani, R. B., and Dymecki, S. M. (2006). Assembly of the brainstem cochlear nuclear complex is revealed by intersectional and subtractive genetic fate maps. Neuron 50, 205-218. doi: 10.1016/j.neuron.2006. 03.014

Floris, A., Diño, M., Jacobowitz, D. M., and Mugnaini, E. (1994). The unipolar brush cells of the rat cerebellar cortex and cochlear nucleus are calretininpositive: a study by light and electron microscopic immunocytochemistry. Anat. Embryol. 189, 495-520. doi: 10.1007/BF00186824

Fredrich, M., Reisch, A., and Illing, R. B. (2009). Neuronal subtype identity in the rat auditory brainstem as defined by molecular profile and axonal projection. Exp. Brain Res. 195, 241-260. doi: 10.1007/s00221-009-1776-7

Friauf, E., and Kandler, K. (1990). Auditory projections to the inferior colliculus of the rat are present by birth. Neurosci. Lett. 120, 58-61. doi: 10.1016/03043940(90)90167-8

Frisina, R. D., Zettel, M. L., Kelley, P. E., and Walton, J. P. (1995). Distribution of calbindin D-28k immunoreactivity in the cochlear nucleus of the young adult chinchilla. Hear. Res. 85, 53-68. doi: 10.1016/0378-5955(95)00 029-4

Fujiyama, T., Yamada, M., Terao, M., Terashima, T., Hioki, H., Inoue, Y. U., et al. (2009). Inhibitory and excitatory subtypes of cochlear nucleus neurons are defined by distinct bHLH transcription factors, Ptfla and Atoh1. Development 136, 2049-2058. doi: 10.1242/dev.033480 
Gallyas, F. (1979). Silver staining of myelin by means of physical development. Neurol. Res. 1, 203-209. doi: 10.1080/01616412.1979.11739553

Gavin, B. J., McMahon, J. A., and McMahon, A. P. (1990). Expression of multiple novel Wnt-1/int-1-related genes during fetal and adult mouse development. Genes Dev. 4, 2319-2332. doi: 10.1101/gad.4.12b.2319

Gurung, B., and Fritzsch, B. (2004). Time course of embryonic midbrain and thalamic auditory connection development in mice as revealed by carbocyanine dye tracing. J. Comp. Neurol. 479, 309-327. doi: 10.1002/cne.20328

Hagan, N., Guarente, J., Ellisor, D., and Zervas, M. (2017). The temporal contribution of the Gbx2 lineage to cerebellar neurons. Front. Neuroanat. 11:50. doi: 10.3389/Fnana.2017.00050

Hagan, N., and Zervas, M. (2012). Wnt1 expression temporally allocates upper rhombic lip progenitors and defines their terminal cell fate in the cerebellum. Mol. Cell. Neurosci. 49, 217-229. doi: 10.1016/j.mcn.2011.11.008

Hayes, L., Zhang, Z., Albert, P., Zervas, M., and Ahn, S. (2011). Timing of sonic hedgehog and Gli1 expression segregates midbrain dopamine neurons. J. Comp. Neurol. 519, 3001-3018. doi: 10.1002/cne.22711

Heizmann, C. W. (1984). Parvalbumin, a relaxing factor in muscle and a neuronal marker in brain. Prog. Clin. Biol. Res. 168, 205-210.

Hippenmeyer, S., Vrieseling, E., Sigrist, M., Portmann, T., Laengle, C., Ladle, D. R., et al. (2005). A developmental switch in the response of DRG neurons to ETS transcription factor signaling. PLoS, Biol. 3:e159. doi: 10.1371/journal.pbio. 0030159

Hurd, L. B., and Feldman, M. L. (1994). Purkinje-like cells in rat cochlear nucleus. Hear. Res. 72, 143-158. doi: 10.1016/0378-5955(94)90214-3

Joyner, A. L., and Zervas, M. (2006). Genetic inducible fate mapping in mouse: establishing genetic lineages and defining genetic neuroanatomy in the nervous system. Dev. Dyn. 235, 2376-2385. doi: 10.1002/dvdy.20884

Kandler, K., and Friauf, E. (1993). Pre- and postnatal development of efferent connections of the cochlear nucleus in the rat. J. Comp. Neurol. 328, 161-184. doi: $10.1002 / \mathrm{cne} .903280202$

Koszeghy, A., Pál, B., Pap, P., Pocsai, K., Nagy, Z., Szucs, G., et al. (2009). Purkinjelike cells of the rat cochlear nucleus: a combined functional and morphological study. Brain Res. 1297, 57-69. doi: 10.1016/j.brainres.2009.08.041

Landsberg, R. L., Awatramani, R. B., Hunter, N. L., Farago, A. F., DiPietrantonio, H. J., Rodriguez, C. I., et al. (2005). Hindbrain rhombic lip is comprised of discrete progenitor cell populations allocated by Pax6. Neuron 48, 933-947. doi: 10.1016/j.neuron.2005.11.031

Lane, P. W. (1967). Swaying. Mouse News Lett. 36:40.

Lee, H. Y., Kleber, M., Hari, L., Brault, V., Suter, U., Taketo, M. M., et al. (2004). Instructive role of $\mathrm{Wnt} / \mathrm{beta}$-catenin in sensory fate specification in neural crest stem cells. Science 303, 1020-1023. doi: 10.1126/science.1091611

Livesey, F. J., and Cepko, C. L. (2001). Vertebrate neural cell-fate determination: lessons from the retina. Nat. Rev. Neurosci. 2, 109-118. doi: 10.1038/3505 3522

Lohmann, C., and Friauf, E. (1996). Distribution of the calcium-binding proteins parvalbumin and calretinin in the auditory brainstem of adult and developing rats. J. Comp. Neurol. 367, 90-109. doi: 10.1002/(SICI)1096-9861(19960325) 367:1<90::AID-CNE7>3.0.CO;2-E

Malmierca, M. S., Saint Marie, R. L., Merchan, M. A., and Oliver, D. L. (2005). Laminar inputs from dorsal cochlear nucleus and ventral cochlear nucleus to the central nucleus of the inferior colliculus: two patterns of convergence. Neuroscience 136, 883-894. doi: 10.1016/j.neuroscience.2005.04.040

Matsunaga, E., Katahira, T., and Nakamura, H. (2002). Role of Lmx1b and Wnt1 in mesencephalon and metencephalon development. Development 129, 5269-5277.

McMahon, A. P., and Bradley, A. (1990). The Wnt-1 (int-1) proto-oncogene is required for development of a large region of the mouse brain. Cell 62, 1073-1085. doi: 10.1016/0092-8674(90)90385-R

McMahon, A. P., Joyner, A. L., Bradley, A., and McMahon, J. A. (1992). The midbrain-hindbrain phenotype of Wnt-1-/Wnt-1- mice results from stepwise deletion of engrailed-expressing cells by 9.5 days postcoitum. Cell 69, 581-595. doi: 10.1016/0092-8674(92)90222-X

Nevue, A. A., Elde, C. J., Perkel, D. J., and Portfors, C. V. (2016). Dopaminergic input to the inferior colliculus in mice. Front. Neurosci. 9:168. doi: 10.3389/ fnana.2015.00168

Nichols, D. H., and Bruce, L. L. (2006). Migratory routes and fates of cells transcribing the Wnt-1 gene in the murine hindbrain. Dev. Dyn. 235, 285-300. doi: $10.1002 /$ dvdy.20611
Osen, K. K. (1970). Course and termination of the primary afferents in the cochlear nuclei of the cat. An experimental anatomical study. Arch. Ital. Biol. 108, 21-51.

Paloff, A. M., Usunoff, K. G., Yotovski, P., Hinova-Palova, D. V., and Ovtscharoff, W. A. (2004). Parvalbumin-like immunostaining in the cat inferior colliculus. Light and electron microscopic investigation. Acta Histochem. 106, 219-234. doi: 10.1016/j.acthis.2003.11.006

Park, J., Cederroth, C. R., Basinou, V., Meltser, I., Lundkvist, G., and Canlon, B. (2016). Identification of a circadian clock in the inferior colliculus and its dysregulation by noise exposure. J. Neurosci. 36, 5509-5519. doi: 10.1523/ JNEUROSCI.3616-15.2016

Paxinos, G. (1999). Chemoarchitectonic Atlas of the Rat Brainstem. San Diego, CA: Academic Press.

Pór, A., Pocsai, K., Rusznák, Z., and Szucs, G. (2005). Presence and distribution of three calcium binding proteins in projection neurons of the adult rat cochlear nucleus. Brain Res. 1039, 63-74. doi: 10.1016/j.brainres.2005. 01.057

Rogers, J. H. (1987). Calretinin: a gene for a novel calcium-binding protein expressed principally in neurons. J. Cell Biol. 105, 1343-1353. doi: 10.1083/jcb. 105.3.1343

Ryugo, D. K., and Parks, T. N. (2003). Primary innervation of the avian and mammalian cochlear nucleus. Brain Res. Bull. 60, 435-456. doi: 10.1016/S03619230(03)00049-2

Sharma, V., Nag, T. C., Wadhwa, S., and Roy, T. S. (2009). Stereological investigation and expression of calcium-binding proteins in developing human inferior colliculus. J. Chem. Neuroanat. 37, 78-86. doi: 10.1016/j.jchemneu. 2008.11.002

Thomas, K. R., and Capecchi, M. R. (1990). Targeted disruption of the murine int1 proto-oncogene resulting in severe abnormalities in midbrain and cerebellar development. Nature 346, 847-850. doi: 10.1038/346847a0

Thomas, K. R., Musci, T. S., Neumann, P. E., and Capecchi, M. R. (1991). Swaying is a mutant allele of the proto-oncogene Wnt-1. Cell 67, 969-976. doi: 10.1016/ 0092-8674(91)90369-A

Vater, M., and Braun, K. (1994). Parvalbumin, calbindin D-28k, and calretinin immunoreactivity in the ascending auditory pathway of horseshoe bats. J. Comp. Neurol. 341, 534-558. doi: 10.1002/cne.9034 10409

Wilkinson, D. G., Bailes, J. A., and McMahon, A. P. (1987). Expression of the proto-oncogene int-1 is restricted to specific neural cells in the developing mouse embryo. Cell 50, 79-88. doi: 10.1016/0092-8674(87) 90664-7

Winer, J. A., and Schreiner, C. E. (2005). "The central auditory system: a functional analysis," in The Inferior Colliculus, ed. S. Winer (New York, NY: Springer), $1-68$.

Yamada, M., Terao, M., Terashima, T., Fujiyama, T., Kawaguchi, Y., Nabeshima, Y., et al. (2007). Origin of climbing fiber neurons and their developmental dependence on Ptfla. J. Neurosci. 27, 10924-10934. doi: 10.1523/JNEUROSCI. 1423-07.2007

Yang, J., Brown, A., Ellisor, D., Paul, E., Hagan, N., and Zervas, M. (2013). Dynamic temporal requirement of Wnt1 in midbrain dopamine neuron development. Development 140, 1342-1352. doi: 10.1242/dev.080630

Zervas, M., Millet, S., Ahn, S., and Joyner, A. L. (2004). Cell behaviors and genetic lineages of the mesencephalon and rhombomere 1. Neuron 43, 345-357. doi: 10.1016/j.neuron.2004.07.010

Zervas, M., and Walkley, S. U. (1999). Ferret pyramidal cell dendritogenesis: changes in morphology and ganglioside expression during cortical development. J. Comp. Neurol. 413, 429-448. doi: 10.1002/(SICI)10969861(19991025)413:3<429::AID-CNE6>3.0.CO;2-7

Conflict of Interest Statement: The authors declare that the research was conducted in the absence of any commercial or financial relationships that could be construed as a potential conflict of interest.

Copyright (c) 2017 Brown and Zervas. This is an open-access article distributed under the terms of the Creative Commons Attribution License (CC BY). The use, distribution or reproduction in other forums is permitted, provided the original author(s) or licensor are credited and that the original publication in this journal is cited, in accordance with accepted academic practice. No use, distribution or reproduction is permitted which does not comply with these terms. 